Article

\title{
Formulation and Optimization of Nanospanlastics for Improving the Bioavailability of Green Tea Epigallocatechin Gallate
}

\author{
Eman A. Mazyed ${ }^{1, *}$, Doaa A. Helal ${ }^{2}$ D, Mahmoud M. Elkhoudary ${ }^{3}$, Ahmed G. Abd Elhameed ${ }^{4,5}$ \\ and Mohamed Yasser 6 \\ 1 Department of Pharmaceutical Technology, Faculty of Pharmacy, Kaferelsheikh University, \\ Kaferelsheikh 33516, Egypt \\ 2 Department of Pharmaceutics, Faculty of Pharmacy, Fayoum University, Fayoum 63514, Egypt; \\ das12@fayoum.edu.eg \\ 3 Department of Pharmaceutical Chemistry, Faculty of Pharmacy, Horus University, \\ New Damietta 34518, Egypt; melkhodary@horus.edu.eg \\ 4 Department of Pharmacology and Toxicology, Faculty of Pharmacy, Mansoura University, \\ Mansoura 35516,Egypt; Ahmed_gamal_helal@mans.edu.eg \\ 5 Department of Pharmacology, Faculty of Pharmacy, Horus University, New Damietta 34518, Egypt \\ 6 Department of Pharmaceutical Technology, Faculty of Pharmacy, Horus University, \\ New Damietta 34518, Egypt; mhamdy@horus.edu.eg \\ * Correspondence: eman_mazyad@pharm.kfs.edu.eg
}

check for updates

Citation: Mazyed, E.A.; Helal, D.A.; Elkhoudary, M.M.; Abd Elhameed, A.G.; Yasser, M. Formulation and Optimization of Nanospanlastics for Improving the Bioavailability of Green Tea Epigallocatechin Gallate. Pharmaceuticals 2021, 14, 68. https://doi.org/10.3390/ph14010068

Received: 18 December 2020

Accepted: 12 January 2021

Published: 15 January 2021

Publisher's Note: MDPI stays neutral with regard to jurisdictional claims in published maps and institutional affiliations.

Copyright: (c) 2021 by the authors. Licensee MDPI, Basel, Switzerland. This article is an open access article distributed under the terms and conditions of the Creative Commons Attribution (CC BY) license (https:/ / creativecommons.org/licenses/by/ $4.0 /)$.

\begin{abstract}
The present study aimed to investigate the potential of nanospanlastics for boosting the bioavailability of epigallocatechin gallate (EGCG). EGCG has valuable effects like anti-inflammation, anti-oxidation, and anti-tumorigenesis. Unfortunately, it has a low oral bioavailability due to its limited permeation and poor stability. To overcome these pitfalls, EGCG was fabricated as a nanospanlastic. Nanospanlastics are flexible nanovesicles that are composed of surfactants and edge activators (EAs). EAs improve the deformability of spanlastics by acting as a destabilizing factor of their vesicular membranes. EGCG-loaded spanlastics were prepared by an ethanol injection method, according to $2^{3}$ factorial design, to explore the impact of different independent variables on entrapment efficiency $(\mathrm{EE} \%), \%$ drug released after $12 \mathrm{~h}\left(\mathrm{Q}_{12 \mathrm{~h}}\right)$, and particle size (PS). In vitro characterization, ex vivo intestinal permeation test, and pharmacokinetic study of the optimized formula were performed. A newly developed RP-HPLC technique was adopted for the estimation of EGCG. The optimized formula (F4) demonstrated more prolonged drug release and a significant improvement in the EE\%, permeability, deformability and stability than the corresponding niosomes. The pharmacokinetic study investigated that F4 had a more sustained drug release and a higher bioavailability than the conventional niosomes and free drugs. Nanospanlastics could be a promising approach for improving the bioavailability of EGCG.
\end{abstract}

Keywords: EGCG; spanlastics; edge activator; bioavailability; optimization

\section{Introduction}

Natural plants are an important and affordable source for valuable drugs that could be used effectively for the treatment of several diseases. Green tea is obtained from tea plant leaves (Camilla sinensis), which are widely used as a traditional health drink and is a vital source of various antioxidant polyphenols [1,2]. Green tea leaves have a number of valuable effects. However, black tea loses these effects as a result of the oxidation of its chemical components [3]. Catechins are the main polyphenolic constituents in green tea that are responsible for the majority of its anti-oxidant effects. Many studies have attributed the various valuable effects of green tea to (-) Epigallocatechin gallate (EGCG) [4,5]. EGCG is the most important green tea flavonoid and has attracted the interest of many researchers 
as a promising drug in the pharmaceutical, nutritional, and cosmetic fields. EGCG has exhibited many pharmacological activities such as anti-tumor, anti-oxidant, and antiinflammatory effects [6-9]. Moreover, it has beneficial effects on cardiovascular diseases, diabetes, Parkinson's disease, stroke, Alzheimer's disease, and obesity [10,11]. However, it has limited bioavailability [12,13], which hinders its pharmacological applications and results in a significant inconsistency between the ex vivo and in vivo studies [13]. The poor bioavailability could be attributed to the difficulty in penetrating the cell membranes due to its high hydrophilicity [14]. Additionally, EGCG is unstable and susceptible to ambient conditions such as $\mathrm{pH}$ changes, oxygen, and other stress factors [13]. Using different formulation strategies for encapsulating EGCG is considered an efficient approach to combat these shortcomings $[1,14,15]$.

The nanovesicular systems, such as liposomes and niosomes, are comprised of polar and non-polar parts; hence they could entrap both hydrophilic and lipophilic drugs. Nanovesicles can also improve both the stability and permeability of encapsulated drugs [2]. Additionally, they could deliver drugs to their target sites in a sustained or controlled manner that enhances their bioavailability [16]. Some studies demonstrate improving the stability and bioavailability of EGCG via encapsulation into nanovesicles. Zou et al. [17] reported that nanoliposomes could efficiently delay the decomposition of EGCG in vitro. Besides, Song et al. [14] explored that the niosomal formulations of $(+)$ catechin and (-) EGCG exhibited higher stability, improved absorption, and lower toxicity than the free drug.

However, these conventional carriers have a non-flexible nature and lack deformability during passage through different biological membranes [18]. Hence, recent studies have discussed improving the deformability of these conventional nanovesicles in order to increase their permeability through different biological membranes [19]. Spanlastics are surfactant-based deformable nanocarriers that were formulated by Kakkar and Kaur [20]. The main components of spanlastics are non-ionic surfactants and EAs. EAs act as a destabilizing factor of the vesicular membranes of nanocarriers increasing their flexibility and permeability across the biological membranes $[19,21]$ by squeezing through different pores of the biological layers without rupture. Nanospanlastics are non-immunogenic, biodegradable, and safe deformable nanovesicles. Moreover, they are more chemically stable than conventional liposomes [22]. Hence, nanospanlastics act as a stable nanocarrier that could encapsulate EGCG and increase its stability. Moreover, spanlastics can improve the poor permeability of EGCG due to their deformability by squeezing through narrow pores of the biological membranes $[18,23]$. The current study aimed to enhance the oral bioavailability of EGCG by encapsulation into spanlastic nanocarriers.

\section{Results and Discussion}

\subsection{HPLC Method Development, Optimization and Validation}

Method development and optimization were based on the achievement of suitable sensitivity, rapid analysis, appropriate peak parameters, and ease of operation in addition to the reasonable chromatographic separation of the studied components from the possible matrix contribution of formulation components [24].

Firstly, methanol was selected as an organic modifier due to its availability and cheaper price with better peak shapes as well [25]. A mobile phase with low proportions of methanol was needed to enhance the separation parameters. A mobile phase with $75 \%$ water and $25 \%$ methanol achieved the best chromatographic characteristics for the ECGC peak with a reasonable retention time. The influence of $\mathrm{pH}$ of the mobile phase on the capacity factor $\left(\mathrm{K}^{\prime}\right)$ of the ECGC peak was investigated in order to optimize HPLC parameters. The impact of $\mathrm{pH}$ was investigated using potassium dihydrogen phosphate buffer at a $\mathrm{pH}$ range of 3-6. $\mathrm{pH} 3$ was chosen in order to improve the ECGC peak shape and to minimize its accompanied asymmetry. Eventually, a flow rate of $1 \mathrm{~mL} / \mathrm{min}$ at an ambient temperature was selected to permit better elution time and reasonable peak separation from the combined matrix components with UV detection at $280 \mathrm{~nm}$. The HPLC 
chromatogram exhibited a sharp peak with obvious baseline separations, Figure 1. The average retention time for ECGC was found to be $5.14 \pm 0.12 \mathrm{~min}$, for seven replicates.

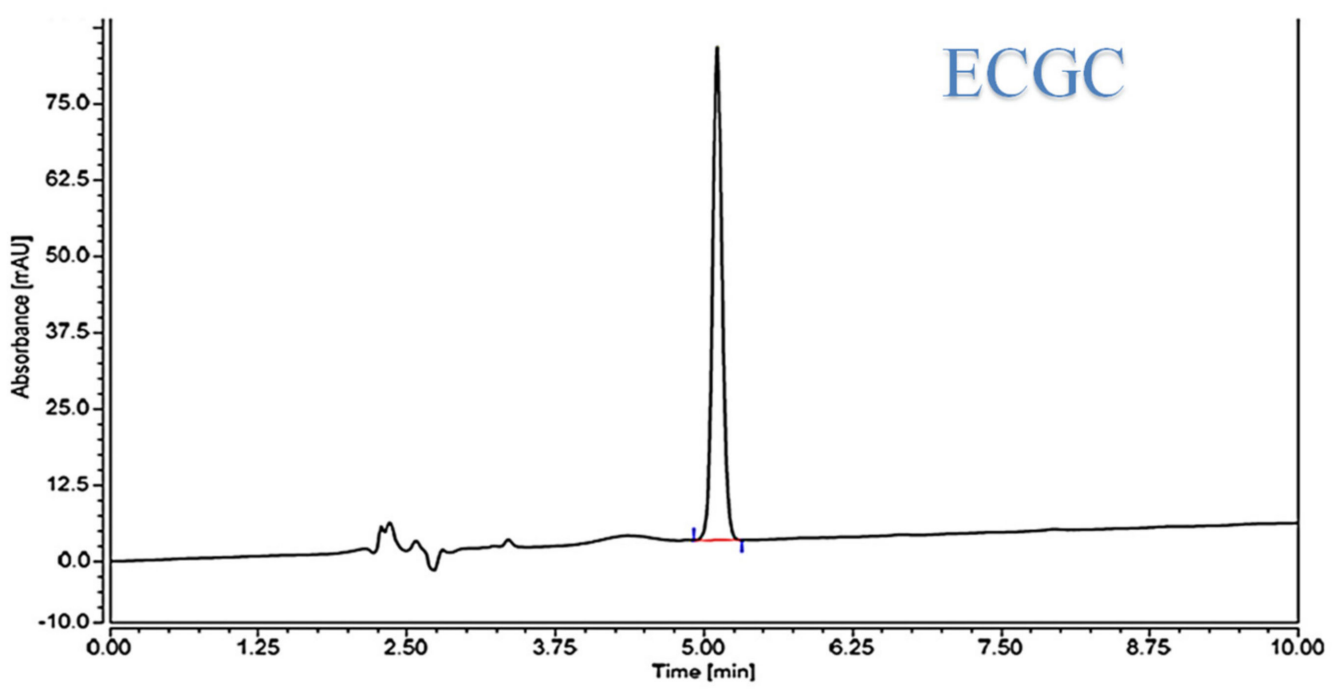

Figure 1. HPLC chromatogram of EGCG.

The optimized method was validated according to the ICH guidelines [26]. The linearity of the current analytical method was granted by the high value of the correlation coefficient (0.999) and the small value of y-intercept (0.0093) and randomly distributed residuals, which demonstrates the linear relationship between the concentrations of EGCG and the peak areas.

The method precision was investigated by the low $(<2 \%)$ coefficients of variation values $(\mathrm{CV} \%)$ for repeatability and intermediate precision studies.

The LOD and LOQ provide an acceptable indication of the sensitivity of the validated technique [27].The detection and quantitation limits were calculated on the basis of the SD of the response approach and they were 35.80 and $108.42 \mathrm{ng} / \mathrm{mL}$, respectively. The system suitability parameters were checked automatically using the Chromeleon 7 software and were found to be within acceptable ranges.

The method specificity was validated by the determination of peak purity factor, with the help of the diode array UV detector (DAD), and was found to be 999. The accuracy of the analytical technique is determined on the basis of the closeness of the obtained values to the true values. The accuracy of the present technique was performed by using known concentrations of ECGC $(300,600$, and $900 \mathrm{ng} / \mathrm{mL})$ and the results were compared with the expected results. The recovery study was conducted in triplicate (for each sample), and the percentage recovery was estimated. The excellent recoveries (95.96-98.12\% with \% RSD of $0.68-1.44 \%$ ) suggested good accuracy of the proposed methods.

Moreover, the robustness of the HPLC method to minor changes in the optimized experimental conditions, such as the flow rate, was studied. The results demonstrated that the method was insensitive to the changes in flow rate. The stability of ECGC was also investigated in the mobile phase. The studied EGCG solutions exhibited no chromatographic changes for 3 days when stored refrigerated at $4{ }^{\circ} \mathrm{C}$ and for $24 \mathrm{~h}$ when kept at room temperature.

The above findings explored that the current optimized and validated HPLC method is a rapid, simple, precise, accurate, and robust technique that can be used for the quantification of EGCG.

\subsection{Preliminary Screening Studies}

The preliminary screening study was used for selecting the most suitable conditions and the most appropriate levels of different independent variables for preparing the spanlastic formulations. The effect of both the rotation speed and the time of sonication on 
EE\% of EGCG-loaded SNVs was studied (Table S1). The results explored that the rotation speed had a significant negative impact on $\mathrm{EE} \%(p<0.01)$ that could be attributed to decreasing the size of EGCG-loaded SNVs by increasing the speed of rotation $[18,28]$.

Additionally, sonication time had a significant negative influence $(p<0.001)$ on $\mathrm{EE} \%$ of EGCG within the nanospanlastic vesicles. Increasing the sonication time to $5 \mathrm{~min}$ resulted in a significant decrease in $\mathrm{EE} \%$ that could be interpreted on the basis of decreasing the size of the SNVs or escaping of EGCG to the external aqueous medium rather than entrapment within the SNVs during disruption and re-aggregation of the nanovesicles by sonication $[29,30]$. Regarding the ratio of non-ionic surfactant to EA, it was observed that the 1:1 ratio had the lowest EE\% $((p<0.01)$, Table S2). These findings could be explained on the basis of increasing the fluidization of the vesicular membrane, which results in increasing drug leakage from the nanospanlastic vesicles at lower concentrations of the non-ionic surfactant [31].

With respect to the type of non-ionic surfactant, Cremophor RH 40 exhibited the lowest EE\% $(p<0.001)$. This may be attributed to its lower lipophilicity (HLB = 14-16) compared to Span $60(\mathrm{HLB}=4.7)$ and Span $80(\mathrm{HLB}=4.3)$, which in turn caused lower vesicle rigidity [32]. Concerning the type of EA, EGCG-loaded spanlastics containing Brij 35 had the lowest EE\% $(p<0.01)$ compared to those containing Tween 60 and Tween 80 . This might be attributable to its higher HLB value (16.9) and the shorter carbon chain length(C12) of Brij 35 [33].

According to the preliminary screening study, 3 min sonication time and $500 \mathrm{rpm}$ rotation speed were selected for the fabrication of EGCG-loaded SNVs using the non-ionic surfactants (Span 60 and Span 80) and the EAs (Tween 80 and Tween 60) at 3:2 and 4:1 ratios of non-ionic surfactants to EA.

\subsection{Analysis of the $2^{3}$ Factorial Design of EGCG-Loaded SNVs}

Table 1 illustrates the composition and the responses (EE\%, $\mathrm{Q}_{12 \mathrm{~h}}$, and PS) of EGCGloaded nanospanlastic formulations prepared according to the $2^{3}$ factorial design. Regression equations demonstrated the influence of various independent variables on the studied responses by comparing the factor coefficients. The positive sign, in front of the factor coefficients, showed a positive influence on the studied responses, whereas the negative sign demonstrated a negative impact $[19,34]$.

Table 1. The $2^{3}$ factorial design and the composition of EGCG-loaded spanlastics.

\begin{tabular}{|c|c|c|c|c|c|c|}
\hline \multirow{3}{*}{ Formula } & \multicolumn{6}{|c|}{ Variables } \\
\hline & \multicolumn{3}{|c|}{ Independent } & \multicolumn{3}{|c|}{ Dependent } \\
\hline & $\mathrm{X} 1$ & $\mathrm{X} 2$ & $\mathrm{X} 3$ & Y1 * & $\mathrm{Y} 2 *$ & $\mathrm{Y} 3$ * \\
\hline F1 & -1 & -1 & -1 & $88.04 \pm 2.82$ & $69.85 \pm 1.33$ & $263.6 \pm 1.62$ \\
\hline $\mathrm{F} 2$ & -1 & -1 & 1 & $92.35 \pm 1.34$ & $62.39 \pm 2.14$ & $229.0 \pm 1.54$ \\
\hline F3 & -1 & 1 & -1 & $70.53 \pm 1.81$ & $92.47 \pm 1.28$ & $318.8 \pm 1.48$ \\
\hline F4 \# & -1 & 1 & 1 & $80.56 \pm 1.57$ & $84.98 \pm 1.33$ & $243.8 \pm 1.24$ \\
\hline F5 & 1 & -1 & -1 & $90.49 \pm 1.22$ & $74.97 \pm 1.36$ & $419.2 \pm 1.82$ \\
\hline F6 & 1 & -1 & 1 & $97.93 \pm 1.28$ & $70.02 \pm 1.25$ & $304.5 \pm 1.55$ \\
\hline F7 & 1 & 1 & -1 & $80.32 \pm 1.38$ & $94.52 \pm 1.07$ & $441.1 \pm 2.03$ \\
\hline F8 & 1 & 1 & 1 & $89.99 \pm 2.16$ & $90.08 \pm 1.46$ & $377.0 \pm 1.93$ \\
\hline \multicolumn{4}{|c|}{ Independent variables } & Low $(-1)$ & \multicolumn{2}{|c|}{$\operatorname{High}(+1)$} \\
\hline \multicolumn{4}{|c|}{ X1: Ratio of Span to EA } & $3: 2$ & \multicolumn{2}{|c|}{$4: 1$} \\
\hline \multicolumn{4}{|c|}{ X2: Type of non-ionic surfactant } & Span 60 & \multicolumn{2}{|c|}{ Span 80} \\
\hline \multicolumn{4}{|c|}{ X3: Type of EA } & Tween 80 & \multicolumn{2}{|c|}{ Tween 60} \\
\hline
\end{tabular}

Notes: Y1: EE (\%), Y2: $\mathrm{Q}_{12 \mathrm{~h}}(\%), \mathrm{PS}(\mathrm{nm}),{ }^{*}$ the values are described as mean $\pm \mathrm{SD}$; all formulations involved 10 mg EGCG, $n=3$, \# optimized Formula. Abbreviations: EE, entrapment efficiency; $\mathrm{Q}_{12 \mathrm{~h}} \%$ drug released after 12 h; PS, Particle Size; EA, edge activator. 
In the present model, adequate precision values for $\mathrm{Y} 1, \mathrm{Y} 2$, and $\mathrm{Y} 3$ were found to be more than 4 (14.698, 32.553, and 15.996, respectively), Table 2. Hence, the chosen model could be used successfully for navigating the design space [23]. The predicted $R^{2}$ was measured to determine the response value predictability. The adjusted $R^{2}$ is the modified version of $R^{2}$ that designates the goodness of fit of the chosen model to the observed data. The values of adjusted and predicted $R^{2}$ statistics should be within about 0.20 of each other in order to be in reasonable harmony [35]. Table 2 demonstrates that the values of $R^{2}$, predicted $R^{2}$ and adjusted $R^{2}$ for different responses are relatively high. Moreover, it is clear that the predicted $R^{2}$ values of different responses were close to the adjusted $R^{2}$ values. These outcomes showed that the obtained data are highly statistically valid and form an excellent fit to the obtained data.

Table 2. The output data of the $2^{3}$ factorial design of EGCG-loaded SNVs.

\begin{tabular}{ccccc}
\hline Responses & $\boldsymbol{R}^{\mathbf{2}}$ & Adjusted $\boldsymbol{R}^{\mathbf{2}}$ & Predicted $\boldsymbol{R}^{\mathbf{2}}$ & Adequate Precision \\
\hline EE\% (Y1) & 0.9502 & 0.9129 & 0.8009 & 14.698 \\
$\mathrm{Q}_{12 \mathrm{~h}}$ (Y2) & 0.9924 & 0.9867 & 0.9695 & 32.553 \\
PS (Y3) & 0.9618 & 0.9331 & 0.8470 & 15.996 \\
\hline
\end{tabular}

Abbreviations: $R^{2}, \mathrm{R}$-squared or the coefficient of determination; $\mathrm{Q}_{12 \mathrm{~h}} \%$ EGCG released after $12 \mathrm{~h}$; EE, entrapment efficiency of EGCG within the SNVs; PS, the particle size of EGCG-loaded SNVs.

Regression equations of different responses in terms of coded factors

$$
\begin{gathered}
\mathrm{EE}(\mathrm{Y} 1)=+86.28+3.40 \times \mathrm{X} 1-5.93 \times \mathrm{X} 2+3.93 \times \mathrm{X} 3 \\
\mathrm{Q} 12 \mathrm{~h}(\mathrm{Y} 2)=+79.91+2.49 \times \mathrm{X} 1+10.60 \times \mathrm{X} 2-3.04 \times \mathrm{X} 3 \\
\mathrm{PS}(\mathrm{Y} 3)=+324.63+60.83 \times \mathrm{X} 1+20.57 \times \mathrm{X} 2-36.04 \times \mathrm{X} 3
\end{gathered}
$$

Additionally, the credibility and the goodness of fit of the current model were investigated from the diagnostic plots for EE\%, $Q_{12}$, and PS of EGCG-loaded SNVs, Figures 2 and 3, respectively. The difference between the actual and predicted values of the three responses was denoted as the residuals. Figures $2 \mathrm{a}, 3 \mathrm{a}$, and $4 \mathrm{a}$ explore the normal probability plots of residuals as a linear pattern with a normal distribution of the residuals. Therefore, the data require no transformation. Figures $2 b, 3 b$, and $4 b$ demonstrate a plot of the residuals versus the predicted responses and show the absence of constant error because the color points, representing the values of different responses, were located within the limits near to the zero-axis and scattered randomly. Figures $2 c, 3 c$, and $4 c$ describe the residual versus run plots and exhibit a random and uniform scattering of points. Hence, no lurking variables affect the tested responses. Figures $2 d, 3 d$, and $4 d$ investigate the excellent analogy between the predicted and the observed values of $Y 1, Y 2$, and $Y 3$ of EGCG-loaded SNVs in the present model.

ANOVA analysis (Table 3) was used to estimate the significance of different independent variables. $p$-value that is lower than the threshold level (0.05) demonstrates the significance of the model terms with rejection of the null hypothesis $\left(\mathrm{H}_{0}\right)$ in favor of the alternative hypothesis. 
a

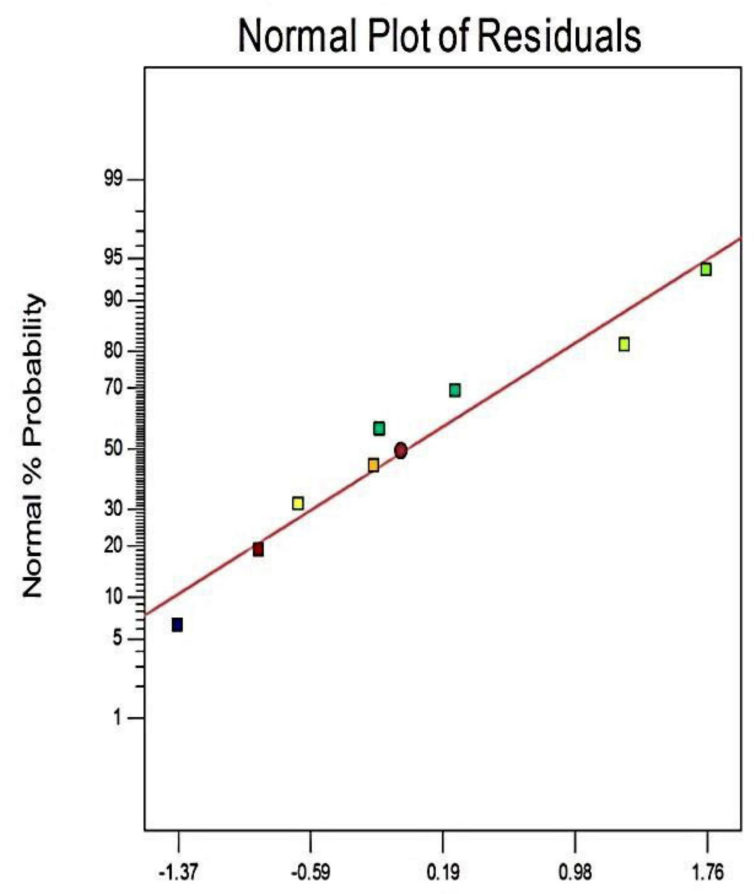

Internally Studentized Residuals

c

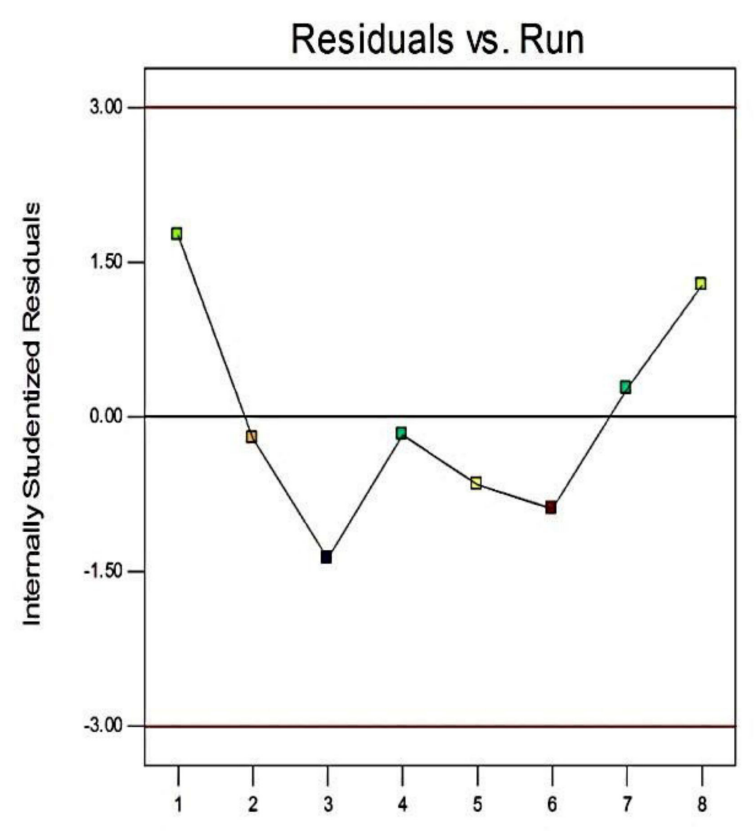

Run Number b

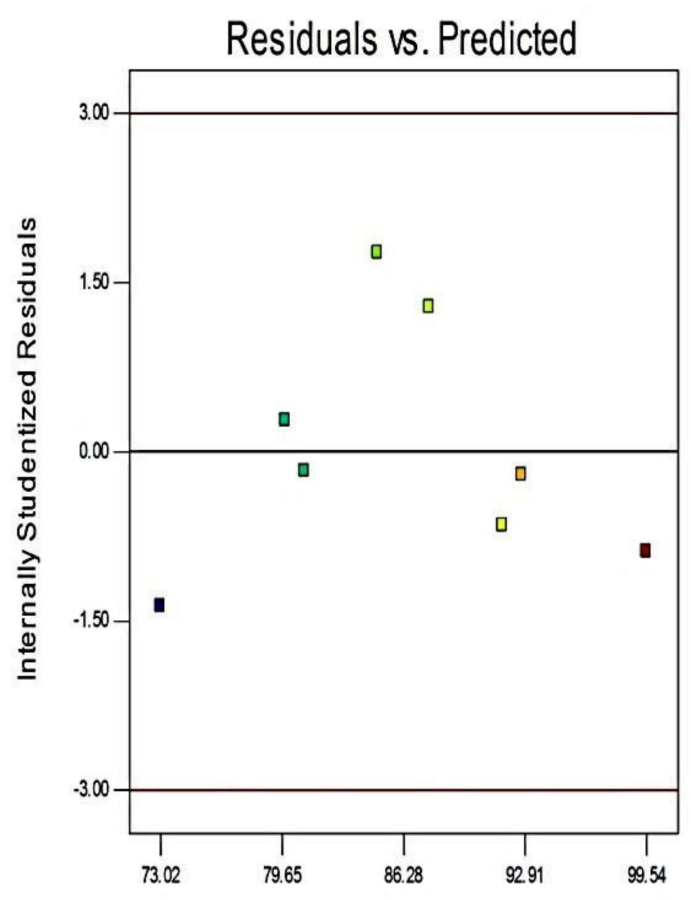

Predicted

d

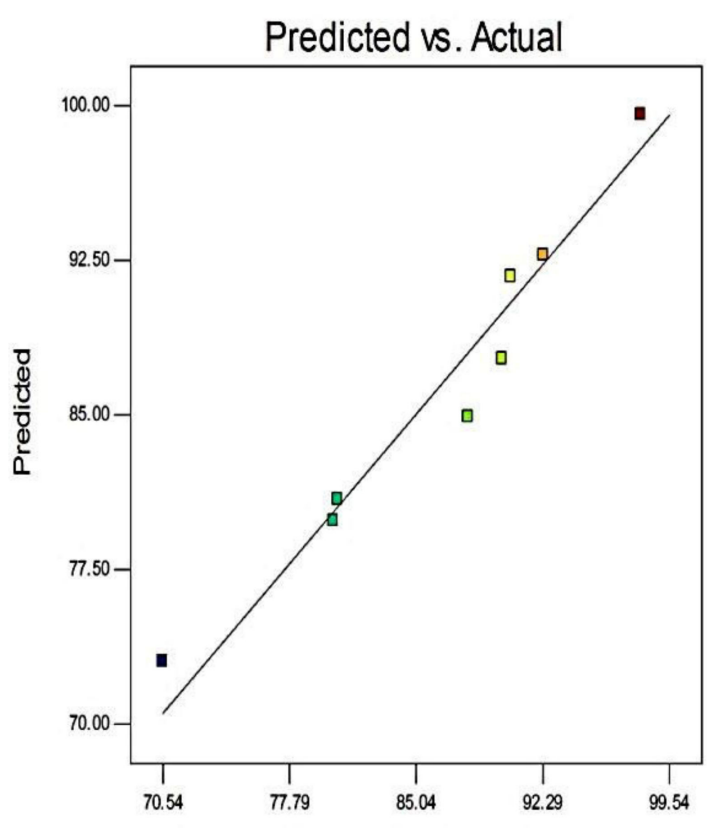

Actual

Figure 2. The diagnostic plots for EE\% of EGCG-loaded spanlastics (a) plot of normal \% probability versus the internally studentized residuals, (b) plot of the internally studentized residuals versus the predicted values, (c) plot of the internally studentized residuals versus the run number, and (d) plot of the predicted versus the actual values of EE\%. Abbreviation: EE, entrapment efficiency of EGCG-loaded spanlastics. 
a
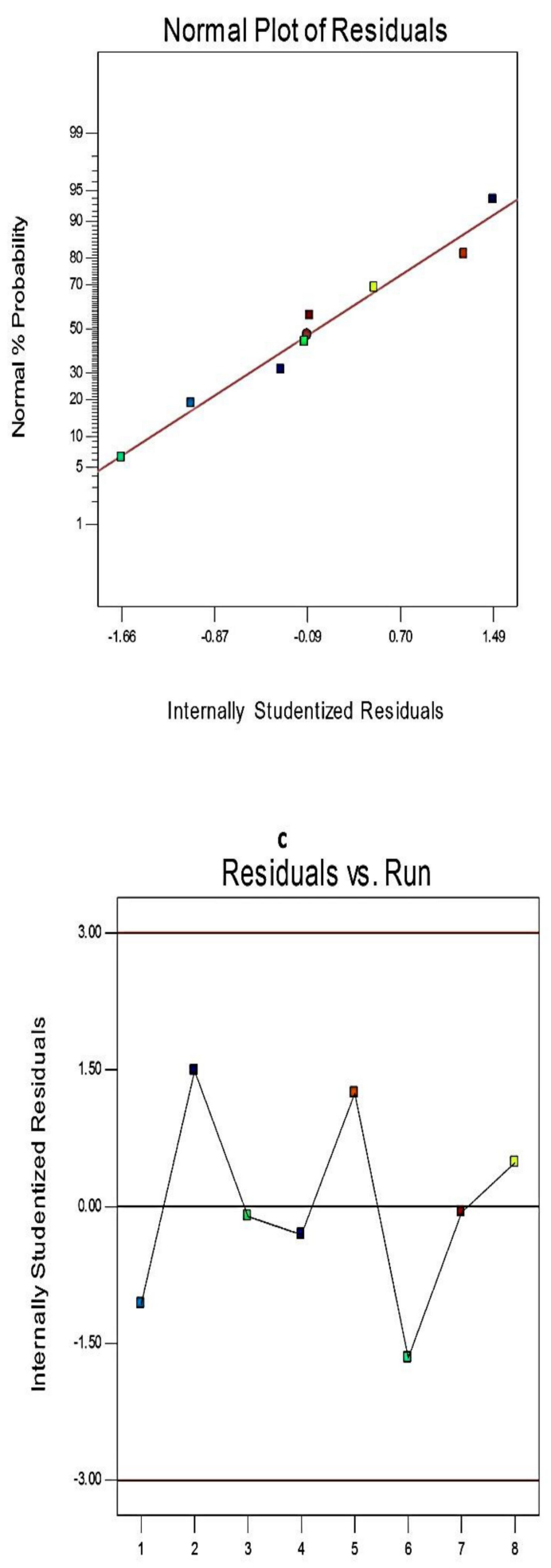

Run Number b
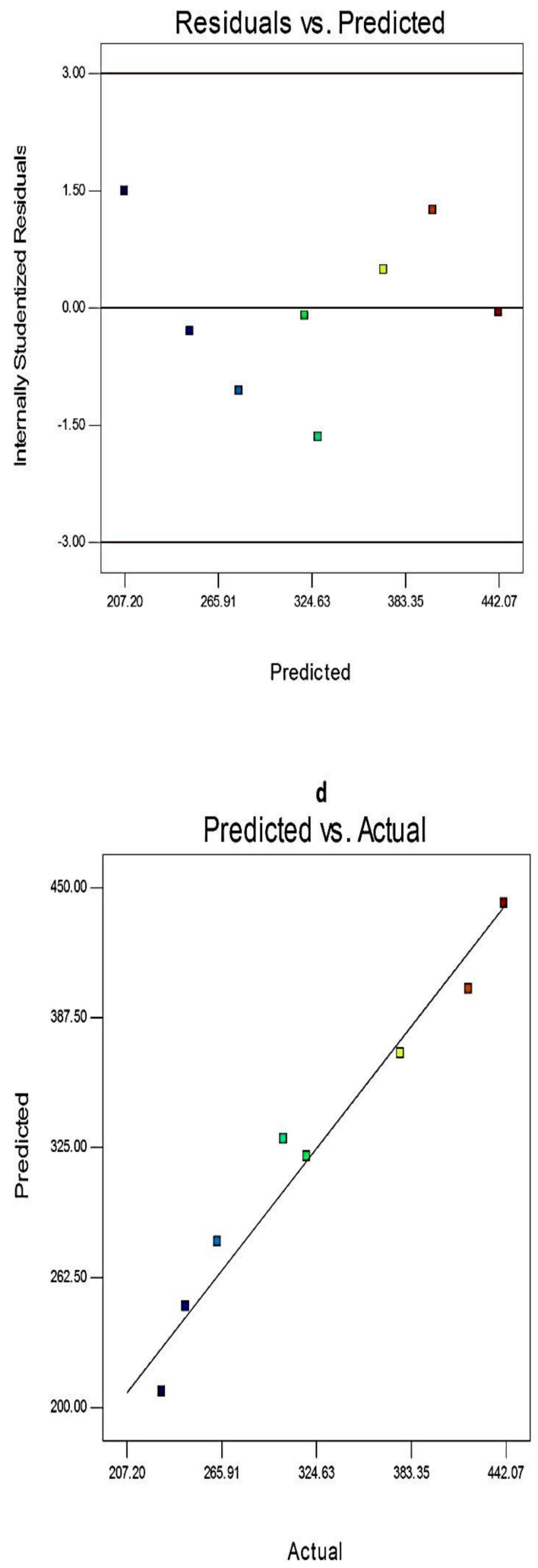

Figure 3. The diagnostic plots for PS of EGCG-loaded spanlastics (a) plot of normal \% probability versus the internally studentized residuals, (b) plot of the internally studentized residuals versus the predicted values, (c) plot of the internally studentized residuals versus the run number, and (d) plot of the predicted versus the actual values. Abbreviation: PS, particle size of EGCG-loaded spanlastics. 
a

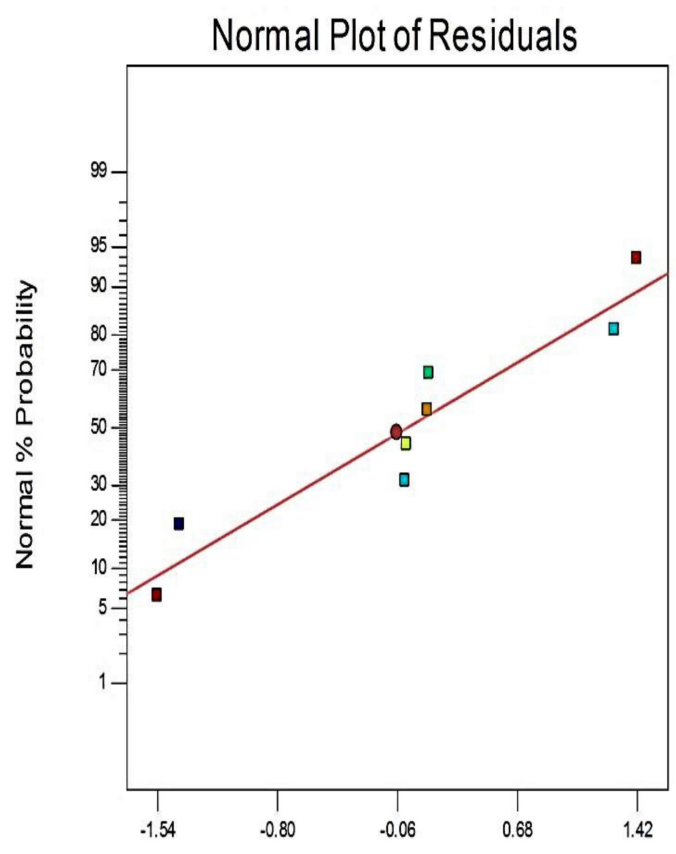

Internally Studentized Residuals

c

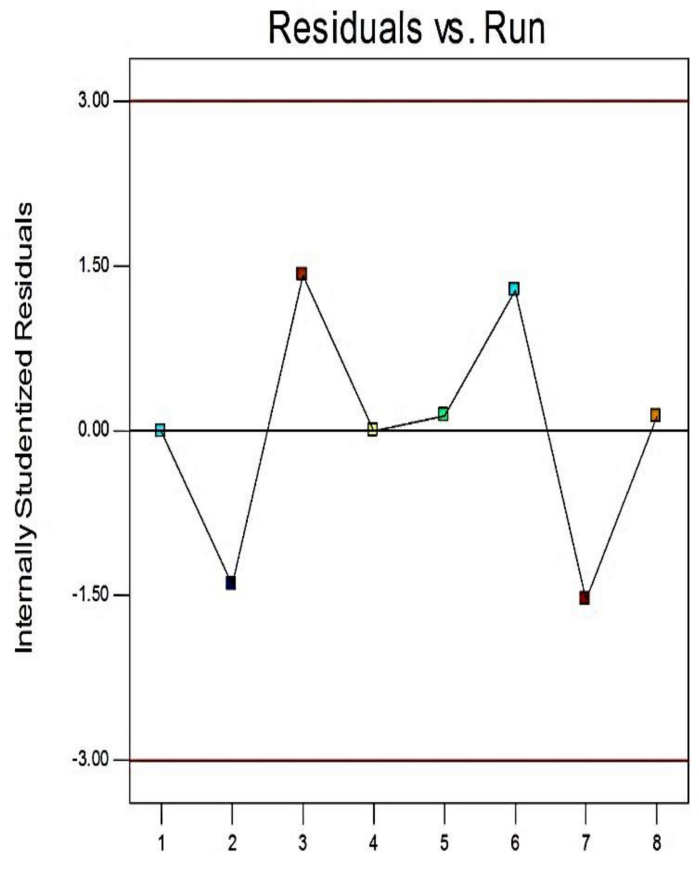

Run Number b
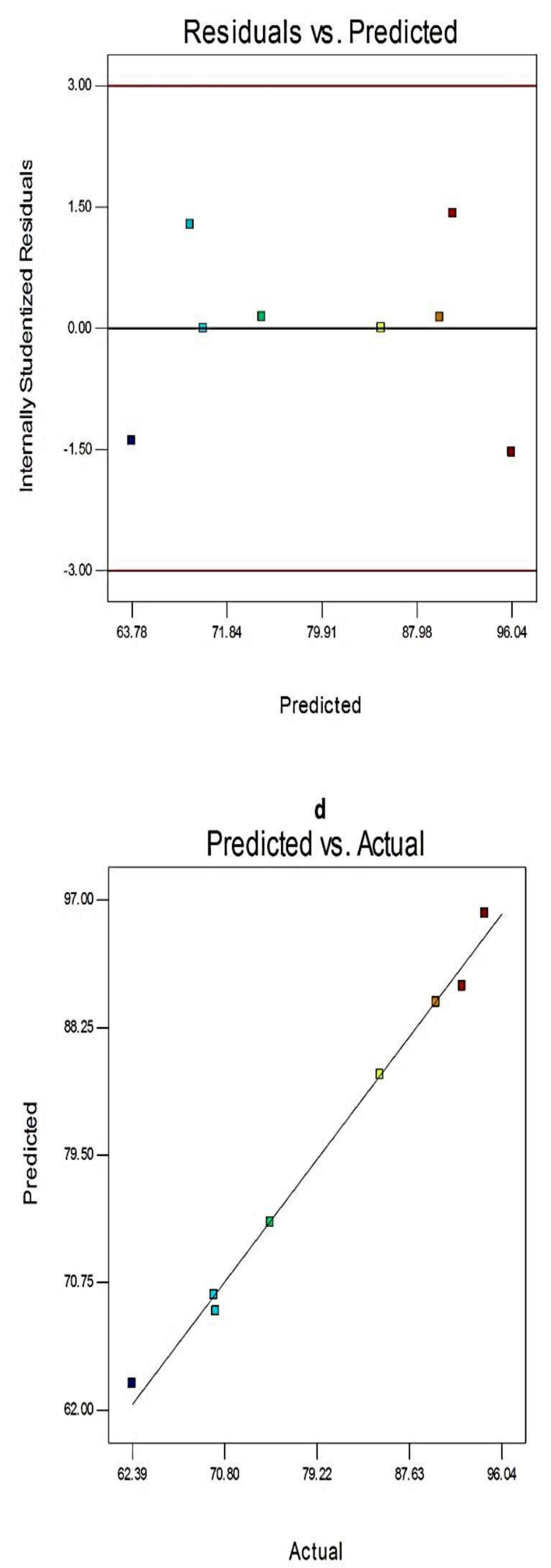

Figure 4. The diagnostic plots for $\mathrm{Q}_{12 \mathrm{~h}}$ of EGCG-loaded spanlastics (a) plot of normal \% probability versus the internally studentized residuals, (b) plot of the internally studentized residuals versus the predicted values, (c) plot of the internally studentized residuals versus the run number, and (d) plot of the predicted versus the actual values of $\mathrm{Q}_{12 \mathrm{~h}}$. Abbreviation: $\mathrm{Q}_{12 \mathrm{~h}}, \%$ EGCG released after $12 \mathrm{~h}$. 
Table 3. ANOVA for the $2^{3}$ factorial design of EGCG-loaded SNVs.

\begin{tabular}{|c|c|c|c|c|c|c|}
\hline $\begin{array}{c}\text { Independent } \\
\text { Variable }\end{array}$ & Source & Sum of Squares & df & Mean Square & F-Value & $p$-Value \\
\hline \multirow{4}{*}{$\mathrm{EE} \%(\mathrm{Y} 1)$} & Model & 497.23 & 31 & 165.74 & 25.45 & 0.0046 \\
\hline & $\mathrm{X} 1$ & 92.66 & 1 & 92.66 & 14.23 & 0.0196 \\
\hline & $\mathrm{X} 2$ & 280.95 & 1 & 280.95 & 43.14 & 0.0028 \\
\hline & X3 & 123.62 & 1 & 123.62 & 18.98 & 0.0121 \\
\hline \multirow{4}{*}{$\mathrm{Q}_{12 \mathrm{~h}}(\mathrm{Y} 2)$} & Model & 1022.56 & 3 & 340.85 & 173.49 & 0.0001 \\
\hline & X1 & 49.54 & 1 & 49.54 & 25.22 & 0.0074 \\
\hline & $\mathrm{X} 2$ & 898.93 & 1 & 898.93 & 457.55 & $<0.0001$ \\
\hline & $\mathrm{X} 3$ & 74.09 & 1 & 74.09 & 37.71 & 0.0036 \\
\hline \multirow{4}{*}{ PS (Y3) } & Model & 43,375 & 3 & 14,458 & 33.30 & 0.0027 \\
\hline & $\mathrm{X} 1$ & 29,598 & 1 & 29,598 & 44.67 & 0.0012 \\
\hline & $\mathrm{X} 2$ & 3383.46 & 1 & 3383.5 & 14.89 & 0.0388 \\
\hline & $\mathrm{X} 3$ & 10,393 & 1 & 10,393 & 40.34 & 0.0080 \\
\hline
\end{tabular}

Notes: Values of $p<0.05$ investigate the significance of model terms, X1: Ratio of Span to EA, X2: Type of non-ionic surfactant, X3: Type of EA. Abbreviations: EE, entrapment efficiency; $\mathrm{Q}_{12 \mathrm{~h}} \%$ drug released after $12 \mathrm{~h}$; PS, particle size; df, degree of freedom.

\subsubsection{The Effect of Formulation Variables on EE\% of EGCG-Loaded SNVs}

The drug content (entrapped + un-entrapped) of EGCG-loaded SNVs ranged from $95.78 \pm 1.22 \%$ to $98.76 \pm 1.18 \%$. EE \% is a vital tool for estimating drug retention within the nanovesicles [36]. EGCG-loaded SNVs exhibited reasonable EE\% that ranged from $70.53 \pm 1.81 \%$ to $97.93 \pm 1.28 \%$, Table 1 .

Figure 5 and Table 3 show the significant effect of the chosen independent variables (the ratio of Span: EA, type of the non-ionic surfactant, and type of EA) on the EE\% of the EGCG-loaded SNVs.

Regarding the influence of the ratio of Span: EA (X1) on EE\%, it is worth noting that $\mathrm{X} 1$ has a significant positive impact $(p<0.05)$ on drug entrapment within the SNVs. That could be attributed to increasing the rigidity of the vesicular membrane and accordingly, minimizing the leakage of the EGCG and improving the EE\% [19].

With respect to the type of non-ionic surfactant (X2), Span 60-based spanlastic formulations have significantly higher EE\% $(p<0.01)$ than Span 80-based SNVs. That could be explained on the basis of the saturated alkyl chain of Span 60 and its higher phase transition temperature $\left(\mathrm{Tc}=53^{\circ} \mathrm{C}\right)$ that result in decreasing the leakage of Span 60-based SNVs. However, Span 80 has a lower phase transition temperature of $-12{ }^{\circ} \mathrm{C}$ [37] and a double bond in its alkyl chain that hamper the development of a tight vesicular membrane [38,39].

In addition, the type of EA (X3) had a significant impact $(p<0.05)$ on EE\% of EGCG within the developed nanospanlastic vesicles. The EE\% of SNVs containing Tween 60 was significantly higher than that of Tween 80 -based nanospanlastic vesicles. That may be attributed to the unsaturation of the Tween 80 alkyl chain that results in increasing both the fluidity of the vesicular membrane and drug leakage [39]. 


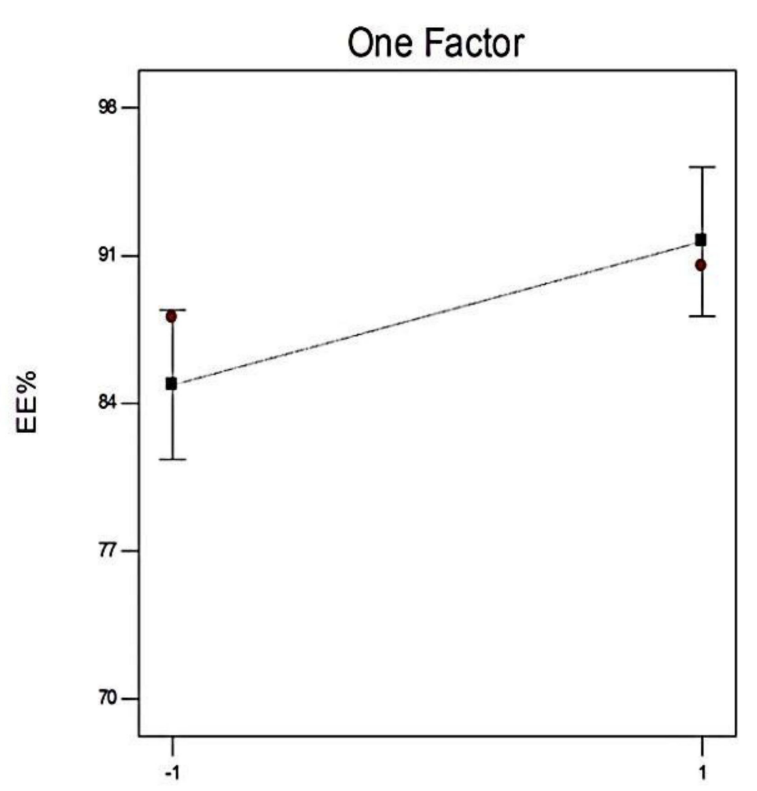

b

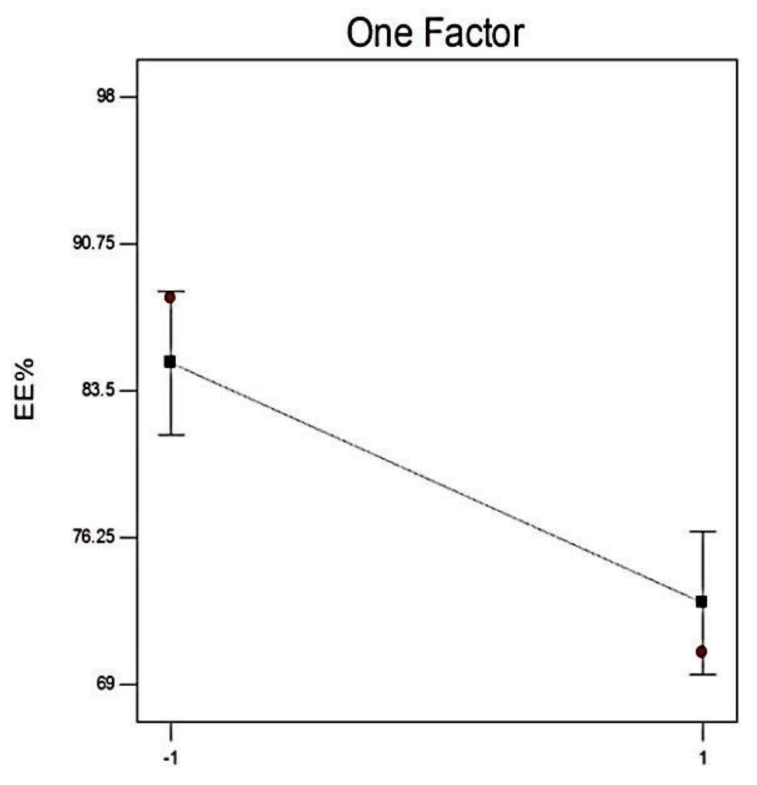

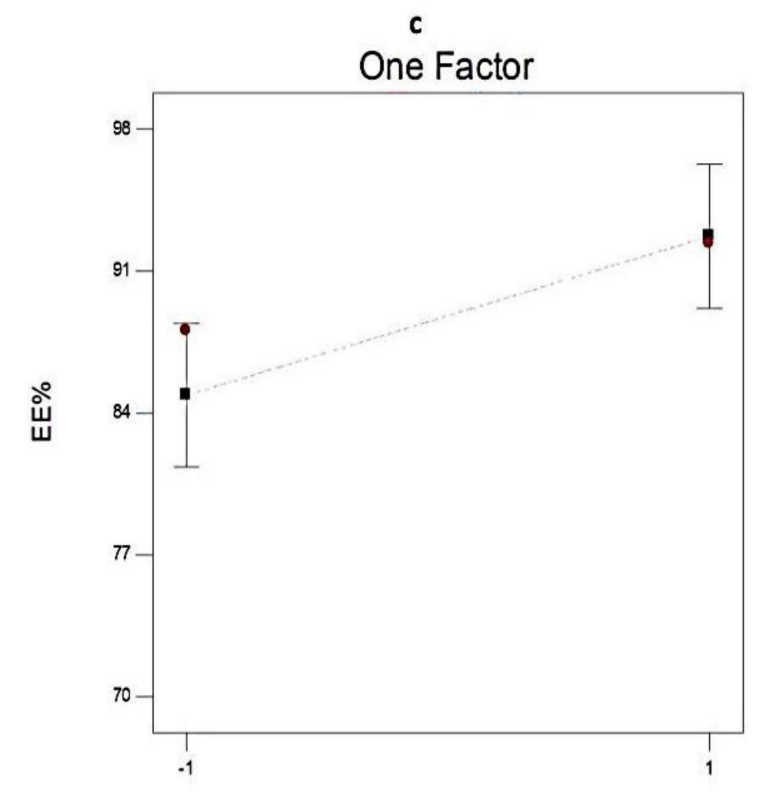

Figure 5. The effect of different independent variables (a) ratio of Span to EA, (b) type of non-ionic surfactant, and (c) type of EA on EE\% of EGCG-loaded spanlastics according to $2^{3}$ factorial design. Abbreviation: EE, entrapment efficiency; EA, edge activator; EGCG, epigallocatechin gallate.

\subsubsection{The Effect of Formulation Variables on $\mathrm{Q}_{12 \mathrm{~h}}$ of EGCG-Loaded Spanlastics}

The sink condition is a critical issue for conducting the in vitro release study [23]. The solubility of EGCG in phosphate-buffered saline $(\mathrm{pH}=7.4)$ was found to be $5.07 \pm 0.21 \mathrm{mg} / \mathrm{mL}$. Therefore, the chosen receptor medium could achieve the required sink conditions because the sink condition is attained when the equilibrium solubility of drugs in the dissolution medium is at least 3 times the volume needed to attain the drug saturation.

The eight EGCG-loaded spanlastic formulations demonstrated a sustained release profile that varied from $62.39 \pm 2.14 \%$ to $94.52 \pm 1.07 \%$ after $12 \mathrm{~h}$ (Figure 6, Table 1). These findings explored that the nanospanlastic vesicles act as perfect reservoirs for EGCG [40]. 
However, the free EGCG exhibited a higher \% drug released of $97.68 \pm 1.33 \%$ after $3 \mathrm{~h}$. That might be attributable to attaining successfully the sink conditions and the absence of hampering the in vitro release of EGCG by the cellulose membrane [41]. These outcomes agreed with Badria et al. [23], who demonstrated that sodium valproate-loaded spanlastics showed a more prolonged drug release than the free drug. Besides, Li et al. [27] reported that the in vitro release of EGCG-loaded niosomal formulations was more sustained than the free drug. Additionally, Safwat et al. [42] demonstrated that free EGCG diffused rapidly throughout the dialysis membrane. By contrast, EGCG-loaded gold nanoparticles exhibited sustained drug release for a prolonged period of time.

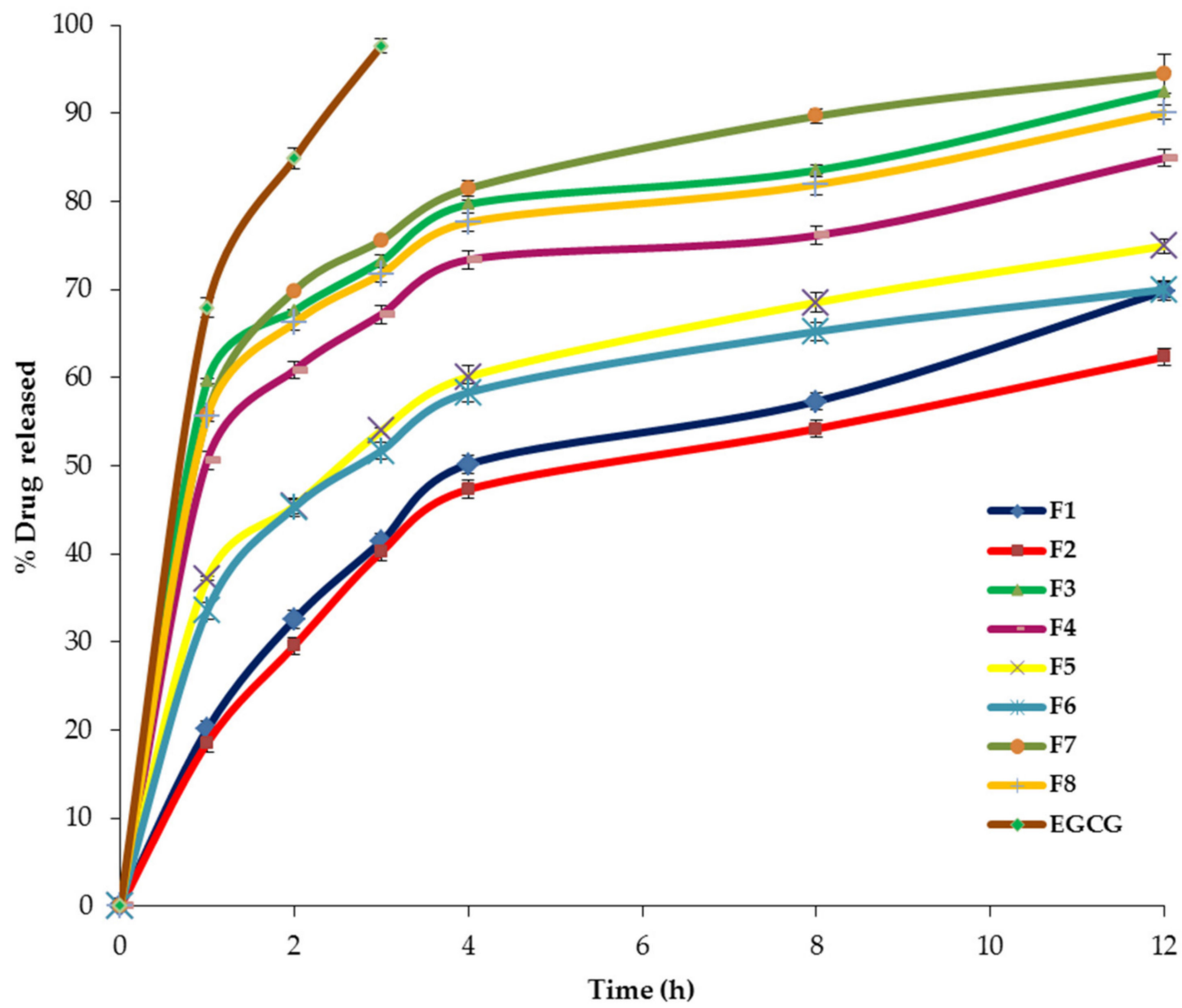

Figure 6. The in vitro release profile of EGCG-loaded spanlastics and EGCG dispersion for $12 \mathrm{~h}$. Abbreviation: EGCG, epigallocatechin gallate.

Figure 7 and Table 3 illustrate the significant impact of different independent variables $(\mathrm{X} 1, \mathrm{X} 2$, and $\mathrm{X} 3)$ on $\mathrm{Q}_{12 \mathrm{~h}}$ of EGCG from SNVs.

The ratio of Span: EA (X1) showed a significant positive impact on $Q_{12 h}$ of the prepared nanospanlastics $(p<0.01)$ that may be due to the formation of mixed micelles, at high levels of EAs, that have a lower sensitivity to the concentration gradient than the vesicles [43].

In addition, it was noted that the $\mathrm{Q}_{12 \mathrm{~h}}$ of Span 80-based SNVs was significantly higher than that in case of Span $60(p<0.0001)$. That could be attributable to the lower phase transition temperature of Span $80\left(\mathrm{Tc}=-12^{\circ} \mathrm{C}\right)$ than Span $60\left(\mathrm{Tc}=53^{\circ} \mathrm{C}\right)$. Hence, Span 60-based vesicles have fewer permeable rigid bilayers than Span 80 . This is also in agreement with the fact that at room temperature, Span 60 molecules are present in the ordered gel state in bilayer structures. However, Span 80 molecules are in the disordered liquid-crystalline state $[44,45]$. 

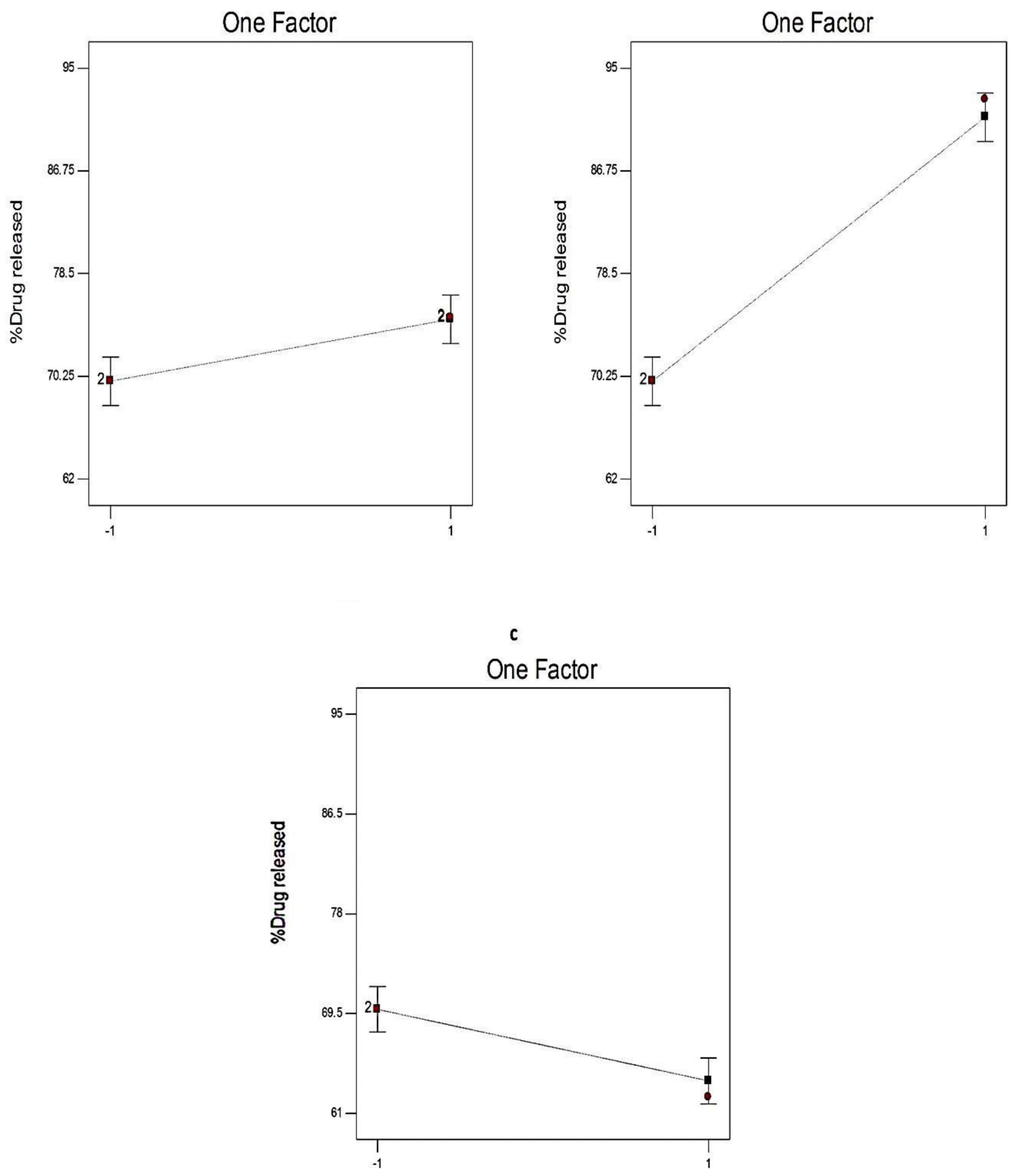

Figure 7. The effect of different independent variables (a) ratio of Span to EA, (b) type of non-ionic surfactant, and (c) type of EA on $\mathrm{Q}_{12 \mathrm{~h}}$ of EGCG-loaded spanlastics according to $2^{3}$ factorial design. Abbreviation: $\mathrm{Q}_{12 \mathrm{~h}} \%$ drug released after $12 \mathrm{~h}$; EA, edge activator; EGCG, epigallocatechin gallate. 
With respect to the type of EA, $\mathrm{Q}_{12 \mathrm{~h}}$ of Tween 80-based SNVs was significantly higher than that in case of Tween $60(p<0.01)$. That may be explained on the basis of the unsaturation of the alkyl chain of Tween 80 that increases the chain fluidity and permeability [39].

According to the kinetic study (Table 4), the in vitro release profile of EGCG-loaded spanlastics fitted well with the Baker-Lonsdale model and that of EGCG dispersion followed by the Higuchi diffusion model, demonstrating a diffusion-based in vitro release mechanism [46]. The Baker-Lonsdale model [47] is established from the Higuchi model [48] to designate the drug release as a diffusion process that is based on the Fickian law.

Table 4. The calculated correlation coefficients for the in vitro release of EGCG and EGCG-loaded spanlastics employing different kinetic orders.

\begin{tabular}{cccccc}
\hline Formula & Zero Order & First Order & Higuchi Model & Hixson Crowell & Baker-Lonsdale \\
\hline F1 & 0.9372 & -0.9740 & 0.9751 & 0.9643 & 0.9867 \\
F2 & 0.9123 & -0.9517 & 0.9612 & 0.9398 & 0.9784 \\
F3 & 0.9365 & -0.9767 & 0.9730 & 0.9728 & 0.9793 \\
F4 & 0.9073 & -0.9595 & 0.9539 & 0.9460 & 0.9725 \\
F5 & 0.9346 & -0.9729 & 0.9765 & 0.9618 & 0.9809 \\
F6 & 0.9493 & -0.9777 & 0.9849 & 0.9697 & 0.9859 \\
F7 & 0.9419 & -0.9888 & 0.9782 & 0.9852 & 0.9920 \\
F8 & 0.9643 & -0.9944 & 0.9904 & 0.9912 & 0.9951 \\
EGCG & 0.9953 & -0.9698 & 0.9998 & 0.9913 & 0.9887 \\
\hline
\end{tabular}

\subsubsection{The Effect of Formulation Variables on PS of EGCG-Loaded SNVs}

Table 1 shows that the PS of EGCG-loaded SNVs ranged between $229.0 \pm 1.54 \mathrm{~nm}$ and $441.1 \pm 2.03 \mathrm{~nm}$. ANOVA results (Table 3) displayed that the ratio of Span: EA (X1), the type of non-ionic surfactant (X2), and the type of EA (X3) significantly affected the PS of the prepared EGCG-loaded SNVs.

The influence of different independent variables $(X 1, X 2$, and $X 3)$ on the PS of the EGCG-loaded SNVs was investigated in Figure 8. Regarding the ratio of Span: EA (X1), the PS of the formulated EGCG-loaded nanovesicles decreased significantly $(p<0.01)$ upon increasing the concentration of EA. That may be attributable to facilitating the partition of particles as a result of reducing the interfacial tension at higher concentrations of EAs [49].

It is clear that the PS of the formulated EGCG-loaded SNVs was significantly $(p<0.05)$ affected by the type of non-ionic surfactant (X2). Span 80-based SNVs are of larger PS than Span 60 nanovesicles. This might be attributed to the development of monolayers of unsaturated Span 80 that are more expanded forming larger molecular areas [50].

The type of EA (X3) has a significant impact on the PS of the prepared EGCG-loaded SNVs. The PS of Tween 80-based SNVs was significantly $(p<0.01)$ larger than that of Tween 60-based vesicles. This could be attributed to the higher hydrophilicity of Tween 80 [51]. Using surfactants of a lower HLB (lower hydrophilicity) resulted in decreasing the surface energy and the development of nanovesicles with a smaller size [52]. These findings are in accordance with Ruckmani and Sankar who reported that Tween 80-based niosomes were larger in size than Tween 60-based vesicles [39] because the nanovesicle size increases as the hydrophilicity of the non-ionic surfactant increases.

The size distribution of the EGCG-loaded nanospanlastics was described in terms of PDI. As the PDI values approach zero, the homogeneity of the colloidal dispersion increases [19]. The prepared nanospanlastic dispersion demonstrated PDI values that ranged from 0.115 to 0.418 , exploring good homogeneity of the EGCG-loaded nanospanlastic dispersions. 


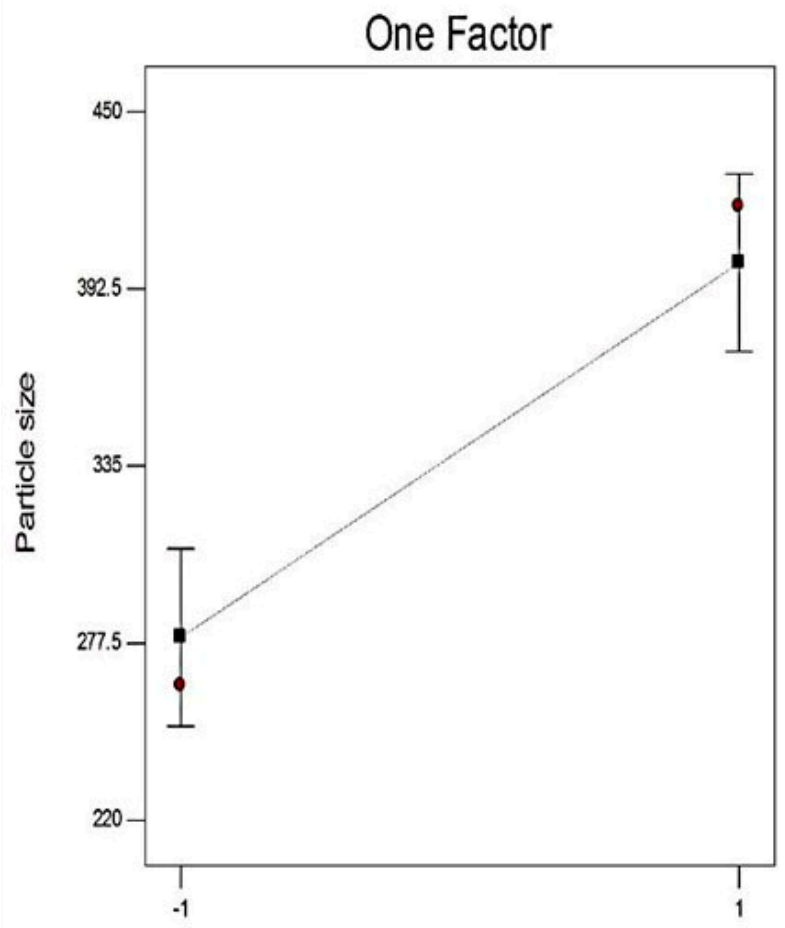

b

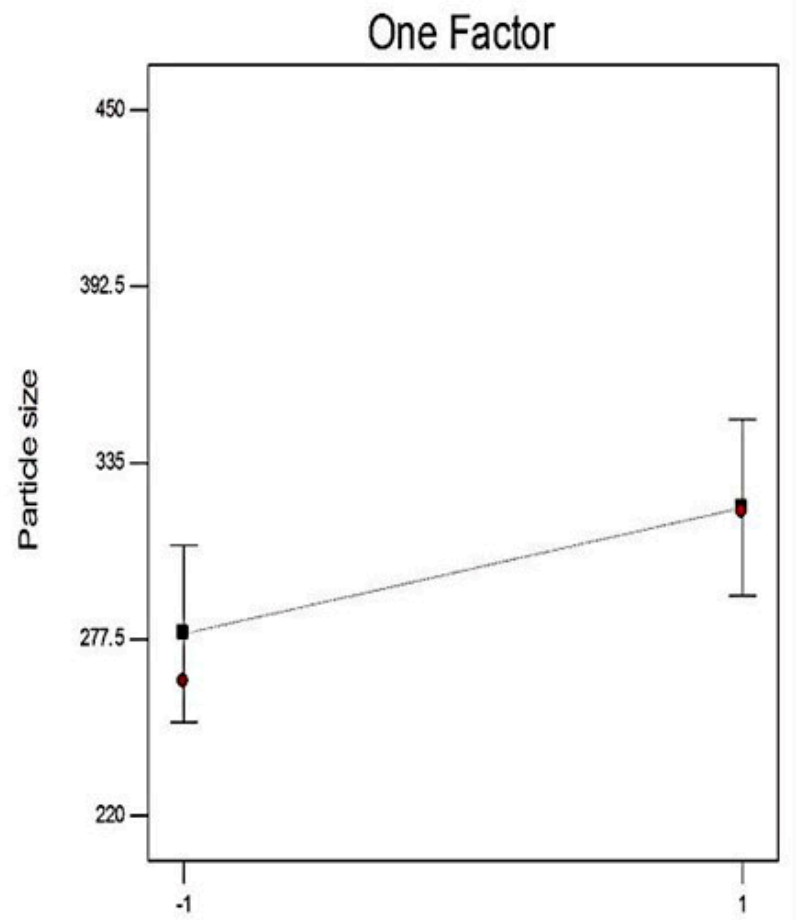

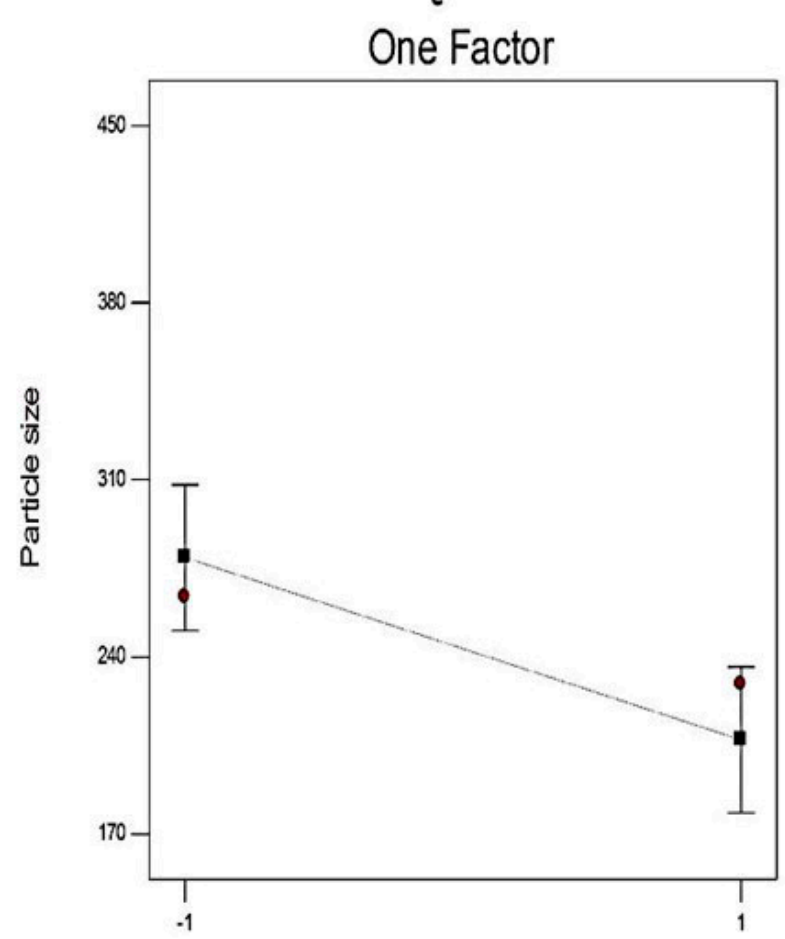

Figure 8. The effect of different independent variables (a) on the ratio of Span to EA, (b) type of non-ionic surfactant, and (c) type of EA on PS of EGCG-loaded spanlastics according to $2^{3}$ factorial design. Abbreviation: PS, particle size; EA, edge activator; EGCG, epigallocatechin gallate. 


\subsubsection{The Optimization of EGCG-Loaded SNVs}

The optimization process is used for the development of systematic strategies to get the best possible combinations required for preparing a high-quality pharmaceutical formula. It involves investigating the effect of different independent variables on the characteristics of pharmaceutical preparations with subsequent selection of the optimized formula [53].

The selection of the optimized EGCG-loaded nanospanlastic formula could be accomplished by means of simultaneous optimization of different variables [54] in the light of the desirability criteria [53]. Each response is described as a desirability value and the overall desirability value is estimated as the average of individual desirability values. The desirability value increases as it becomes closer to the target value [23,54]. The eight EGCG-loaded nanospanlastic formulations were optimized for the EE\% (Y1), $\mathrm{Q}_{12 \mathrm{~h}}(\mathrm{Y} 2)$, and PS (Y3). The optimization process aimed to maximize both $\mathrm{EE} \%$ and $\mathrm{Q}_{12 \mathrm{~h}}$ and minimize PS. The results demonstrated that $\mathrm{F} 4$ had the highest desirability value. Besides, the predicted values of $\mathrm{F} 4$ were $80.88 \%, 84.98 \%$, and 248.33 for $Y 1, Y 2$, and $Y 3$, respectively. The fitness of the current model, in choosing the optimized formula, was also investigated by the small percentage relative error $\left(0.39,-0.002\right.$, and 1.82) for $\mathrm{EE} \%, \mathrm{Q}_{12 \mathrm{~h}}$, and PS, respectively of $\mathrm{F} 4$ [31]. As a result, F4 could be considered as the optimized EGCG-loaded nanospanlastic formula.

\subsection{Comparative Study of the Optimized EGCG-Loaded SNVs and the Conventional Niosomes}

\subsubsection{Determination of EE\%}

The \%EE of the optimized EGCG-loaded spanlastic formula $(80.56 \pm 1.57 \%)$ was significantly $(p<0.01)$ higher than the corresponding niosomal formula that has EE\% of $69.84 \pm 1.35 \%$. These results are in accordance to Kakkar et al. [20] who found that the Ketoconazole-loaded spanlastic formula had a significantly higher EE\% (68.82\%) than the conventional niosomes (35.05\%).

\subsubsection{In Vitro Release Study}

Figure 9 demonstrates the in vitro release profiles of EGCG from the optimized spanlastic formula ( $\mathrm{F} 4)$ and the corresponding niosomal dispersion. It is clear that the in vitro release from optimized EGCG-loaded SNVs $(84.98 \pm 1.33 \%$ after $12 \mathrm{~h}$ ) was more sustained than that from the conventional niosomal formula that exhibited $97.11 \pm 1.24 \%$ drugs released after $8 \mathrm{~h}$. That could be interpreted on the basis of better entrapment of EGCG within the SNVs. These findings are in agreement with ElMeshad et al. [52] who found that different Itraconazole-loaded spanlastic formulations exhibited more sustained drug release than the conventional niosomes. Both the spanlastic and the niosomal formulations demonstrated more sustained drug release than free EGCG dispersion that exhibited $97.68 \pm 1.33 \%$ drugs released after $3 \mathrm{~h}$. 


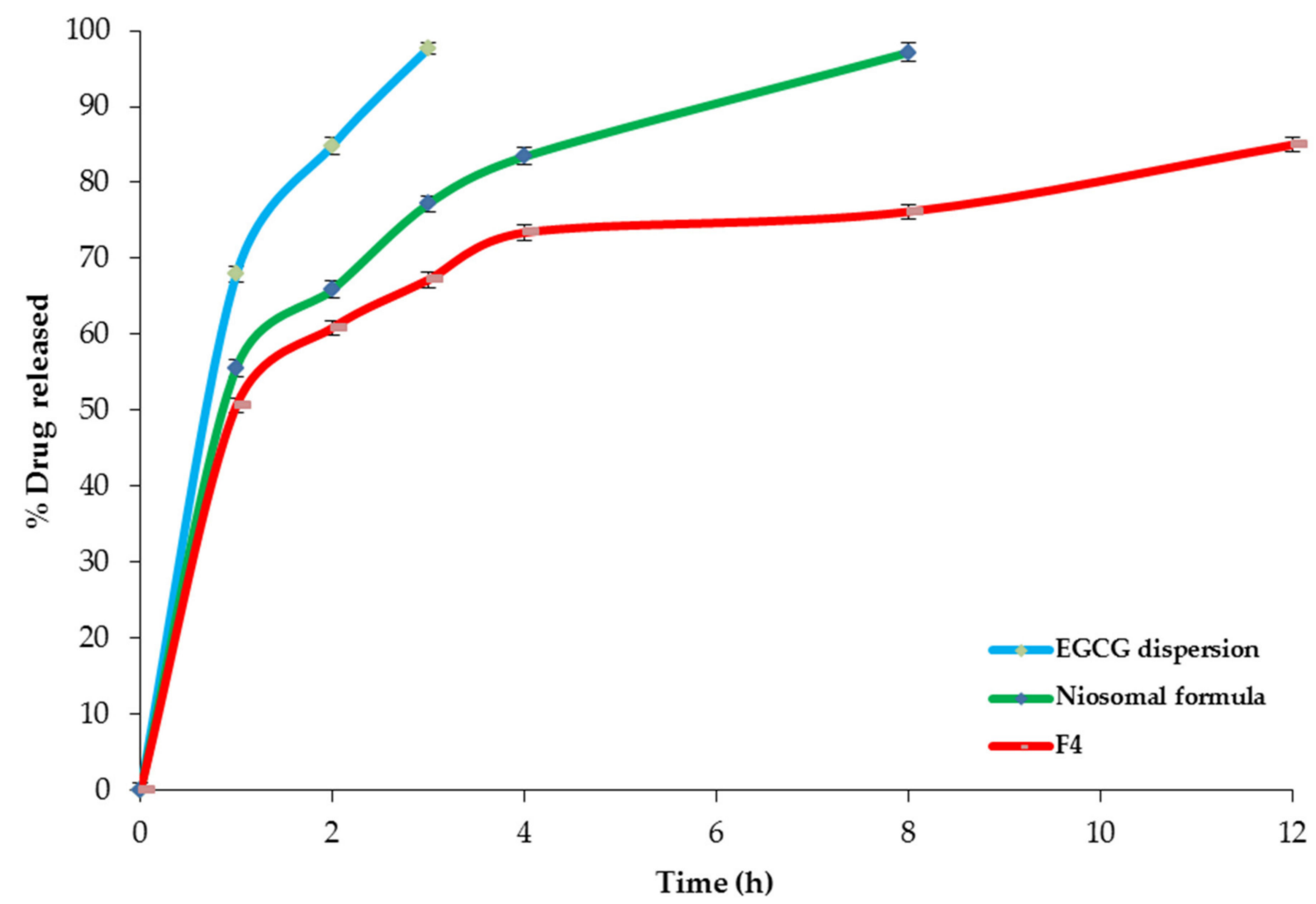

Figure 9. The in vitro release profile of F4, the conventional niosomes, and EGCG dispersion. Abbreviation: EGCG, epigallocatechin gallate.

\subsubsection{Ex Vivo Intestinal Permeation Study}

The ex vivo intestinal permeation study investigated the effect of encapsulation of EGCG within the spanlastic and the niosomal nanovesicles on the permeation through the excised rat intestine. Table 5 and Figure 10 explore the permeation of EGCG from the optimized spanlastic formula (F4) and the niosomal formula in comparison with free EGCG dispersion. It is obvious that the optimized spanlastic formula (F4) showed significantly higher \% drug permeated $(62.22 \pm 1.23 \%)$ than both the niosomal $(50.22 \pm 1.17 \%)$ and EGCG dispersion $(24.77 \pm 0.92 \%)(p<0.05$ and $p<0.01$, respectively) after $12 \mathrm{~h}$. Moreover, Table 5 shows a significant enhancement of EGCG flux from both the optimized spanlastic formula and the niosomal formula than EGCG dispersion, with enhancement ratios of 6.76 and 4.73 , respectively.

Table 5. Ex vivo permeation parameters of F4, the conventional niosomes, and EGCG dispersion.

\begin{tabular}{cccc}
\hline Formula & $\begin{array}{c}{ }^{*} \mathbf{J}_{\mathbf{s s}} \\
\left(\boldsymbol{\mu} \mathbf{~ c m}^{-\mathbf{2}} \mathbf{h}^{-\mathbf{1}}\right)\end{array}$ & $\begin{array}{c}{ }^{*} \mathbf{K}_{\mathbf{P}} \\
\left(\mathbf{c m ~ h} \mathbf{~}^{-\mathbf{1}}\right)\end{array}$ & ER \\
\hline EGCG dispersion & $1.35 \pm 0.11$ & $0.0034 \pm 0.12$ & $-1 .--$ \\
Niosomal formula & $6.38 \pm 1.23$ & $0.0119 \pm 0.14$ & 4.73 \\
F4 & $9.13 \pm 1.34$ & $0.0228 \pm 0.13$ & 6.76
\end{tabular}

Notes: ${ }^{*}$ Each value represents average $\pm \mathrm{SD}(n=3)$, F4: the optimized EGCG-loaded SNVs. Abbreviations: $\mathrm{J}_{\mathrm{ss}}$ the steady-state flux; $\mathrm{K}_{\mathrm{P}}$, the permeability coefficient; ER, Enhancement ratio. 


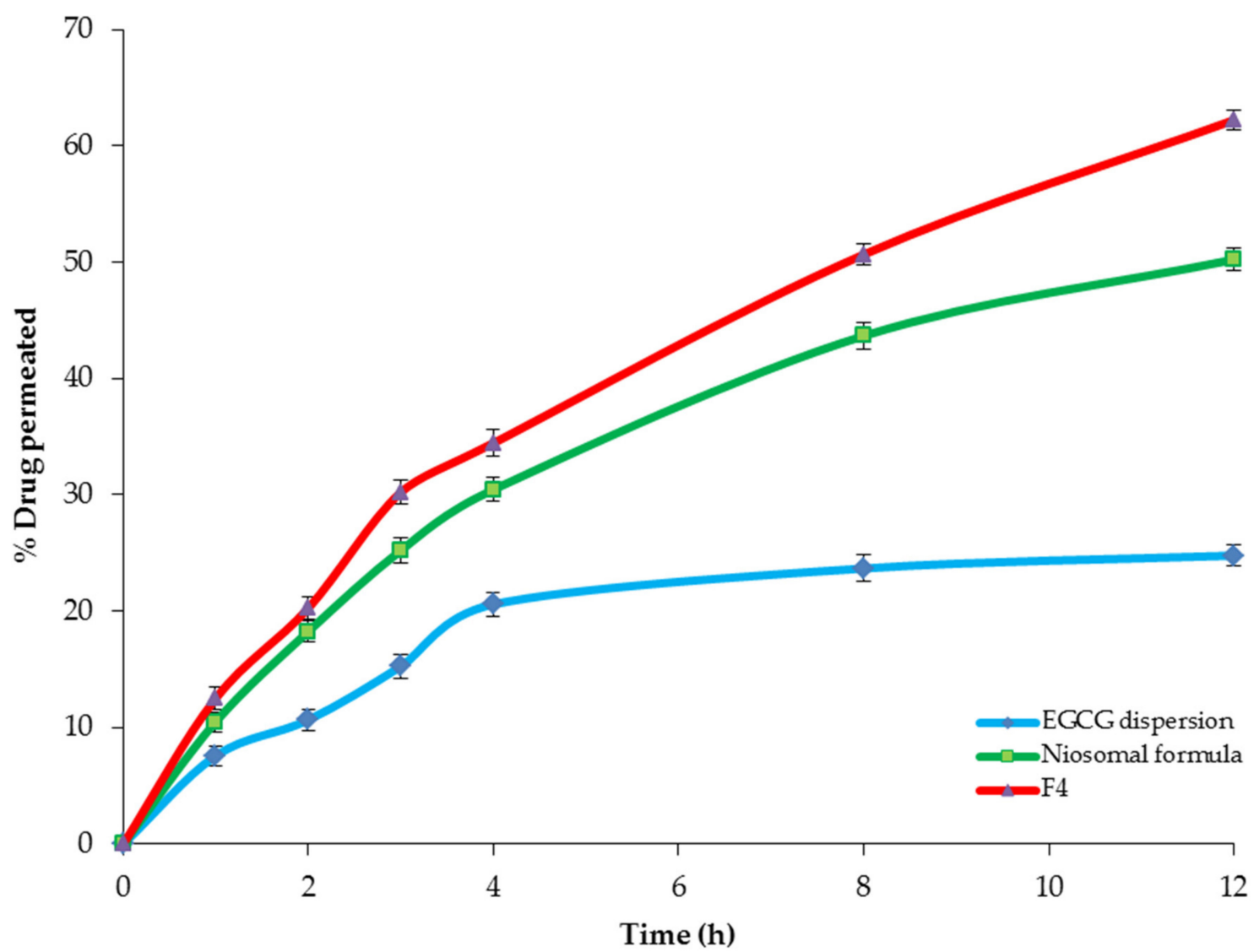

Figure 10. The ex vivo permeation profile of F4, the conventional niosomes, and EGCG dispersion for $12 \mathrm{~h}$. Abbreviation: EGCG, epigallocatechin gallate..

The higher permeation of EGCG-loaded SNVs could be explained on the basis of the higher deformability of spanlastic vesicles due to the presence of the EAs that increase the permeability of the SNVs across different biological membranes by squeezing through their narrow pores without rupture [55]. Additionally, the SNVs act as permeation enhancers that increase the drug permeability through biological membranes [35].

The kinetic analysis investigated that the permeation profiles of F4 and the niosomal formula followed the Baker-Lonsdale model, whereas EGCG dispersion followed the Higuchi model, Table 6.

Table 6. The calculated correlation coefficients for the ex vivo permeation of F4, the niosomal formula, and EGCG employing different kinetic orders.

\begin{tabular}{cccccc}
\hline Formula & Zero Order & First Order & Higuchi Model & Hixson Crowell & Baker-Lonsdale \\
\hline F4 & 0.9779 & -0.9954 & 0.9974 & 0.9911 & 0.9985 \\
Niosomal & 0.9642 & -0.9820 & 0.9931 & 0.9768 & 0.9970 \\
formula & 0.8841 & -0.8930 & 0.9396 & 0.8901 & 0.9263 \\
EGCG & & & &
\end{tabular}

\subsubsection{Measurement of Vesicle Deformability}

The elasticity of the spanlastic vesicles describes their ability to permeate and squeeze through the narrow pores of biological membranes without rupture. This property distinguishes them from conventional niosomes [23]. The DI of the optimized EGCG-loaded SNVs $(19.27 \pm 1.2)$ was significantly $(p<0.0001)$ higher than that $(1.48 \pm 0.02)$ of the corresponding niosomes. This could be explained on the basis of increasing the flexibility of the lipid bilayer [19] by the addition of EAs. 
The vesicle size of the spanlastic and the niosomal formulations was estimated before and after extrusion (Table 7). It is clear that EGCG-loaded SNVs retained their size after extrusion with no significant difference $(p>0.05)$ in the vesicle size. This could be attributed to increasing the deformability of the spanlastic vesicles after the addition of EA [56,57]. However, the significant decrease $(p<0.0001)$ in the vesicle size of EGCG-loaded niosomes, after extrusion, may be explained on the basis of the lack of deformability of the niosomal vesicles which results in rupturing the nanovesicles during passage through the nylon membrane [58].

Table 7. Determination of the elasticity of EGCG-loaded SNVs and the corresponding niosomes.

\begin{tabular}{cccc}
\hline Formula & PS before Extrusion $(\mathbf{n m})$ & PS after Extrusion $(\mathbf{n m})$ & DI \\
\hline F4 & $243.8 \pm 1.24$ & $240.9 \pm 1.27$ & $19.27 \pm 1.2$ \\
Niosomes & $287.4 \pm 1.52$ & $58.2 \pm 1.14$ & $1.48 \pm 0.02$ \\
\hline
\end{tabular}

Notes: F4, the optimized EGCG-loaded SNVs; the values are presented as mean \pm SD; $n=3$. Abbreviations: DI deformability index; PS, particle size.

\subsubsection{The Stability Study}

According to the results of the stability test, it is obvious that the stored EGCG-loaded spanlastic formula (F4) demonstrated no significant difference $(p>0.05)$ in the EE\%, drug content, $\mathrm{Q}_{12 \mathrm{~h}}, \%$ drug permeated, and DI upon comparing with the fresh spanlastic formula (Table 8 ). Whereas, the stored niosomal formula exhibited a significant decrease in the drug content $(p<0.01), \mathrm{EE} \%(p<0.01), \mathrm{Q}_{12 \mathrm{~h}}(p<0.001), \%$ drug permeated $(p<0.01)$, and DI $(p<0.001)$ when compared to the fresh formula. These results showed that nanospanlastics are more stable carriers than conventional niosomes. This could be explained on the basis of better drug encapsulation within the SNVs.

Table 8. Effect of storage at $4-8{ }^{\circ} \mathrm{C}$ on the properties of the EGCG-loaded spanlastic formula (F4) and the corresponding niosomal formula.

\begin{tabular}{ccccc}
\hline \multirow{2}{*}{ Parameter } & \multicolumn{2}{c}{ Spanlastic Formula } & \multicolumn{2}{c}{ Niosomal Formula } \\
\cline { 2 - 5 } & Fresh & Stored & Fresh & Stored \\
\hline Drug content (\%) & $98.22 \pm 1.21$ & $97.98 \pm 1.14$ & $99.14 \pm 0.82$ & $90.22 \pm 1.60$ \\
EE (\%) & $80.56 \pm 1.57$ & $78.88 \pm 1.18$ & $69.84 \pm 1.20$ & $60.22 \pm 1.57$ \\
$\mathrm{Q}_{12 \mathrm{~h}}(\%)$ & $84.98 \pm 1.14$ & $83.74 \pm 0.88$ & $97.11 \pm 1.24$ & $85.92 \pm 1.37$ \\
\% drug permeated & $62.22 \pm 1.23$ & $61.05 \pm 1.33$ & $50.22 \pm 1.17$ & $39.56 \pm 1.22$ \\
DI & $19.27 \pm 0.92$ & $18.81 \pm 0.72$ & $1.48 \pm 0.12$ & $0.69 \pm 0.05$
\end{tabular}

Notes: Each value is described as mean $\pm \mathrm{SD}(n=3)$; F4, the optimized EGCG-loaded spanlastics. Abbreviations: $\mathrm{EE}$, entrapment efficiency; $\mathrm{Q}_{12 \mathrm{~h}}, \%$ drug released after $12 \mathrm{~h}$; DI, deformability index.

\subsection{Characterization of the Optimized EGCG-Loaded Spanlastic Formula}

\subsubsection{Morphological Characterization by SEM}

The optimized EGCG-loaded nanospanlastic formula (F4) appeared as a homogenous dispersion of well-identified spherical vesicles (Figure 11). It is possible to interpret the spherical shape of EGCG-loaded SNVs on the basis of the amphiphilicity of the nonionic surfactants that results in their tendency to decrease the surface-free energy and spontaneous development of spherical nanospanlastic vesicles within the aqueous environment $[59,60]$. 


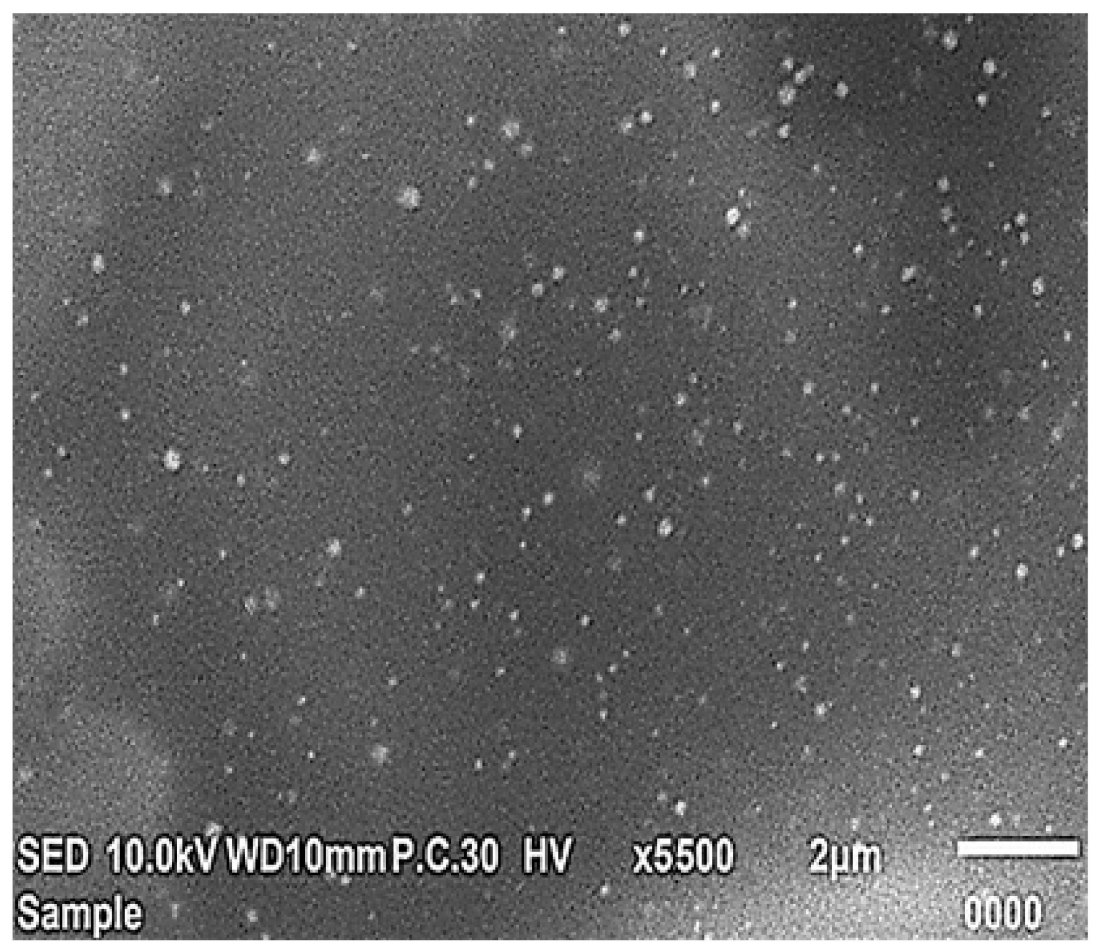

Figure 11. Scanning electron micrograph of the optimized EGCG-loaded nanospanlastic formula (F4). Abbreviation: EGCG, epigallocatechin gallate.

\subsubsection{Determination of Particle Size and Zeta Potential}

The particle size of the optimized EGCG-loaded SNVs (F4) was found to be $243.8 \pm 1.24 \mathrm{~nm}$, with a PDI value of 0.21 . The small value of PDI reveals the homogenous distribution of the particle size of EGCG-loaded SNVs, Figure 12.

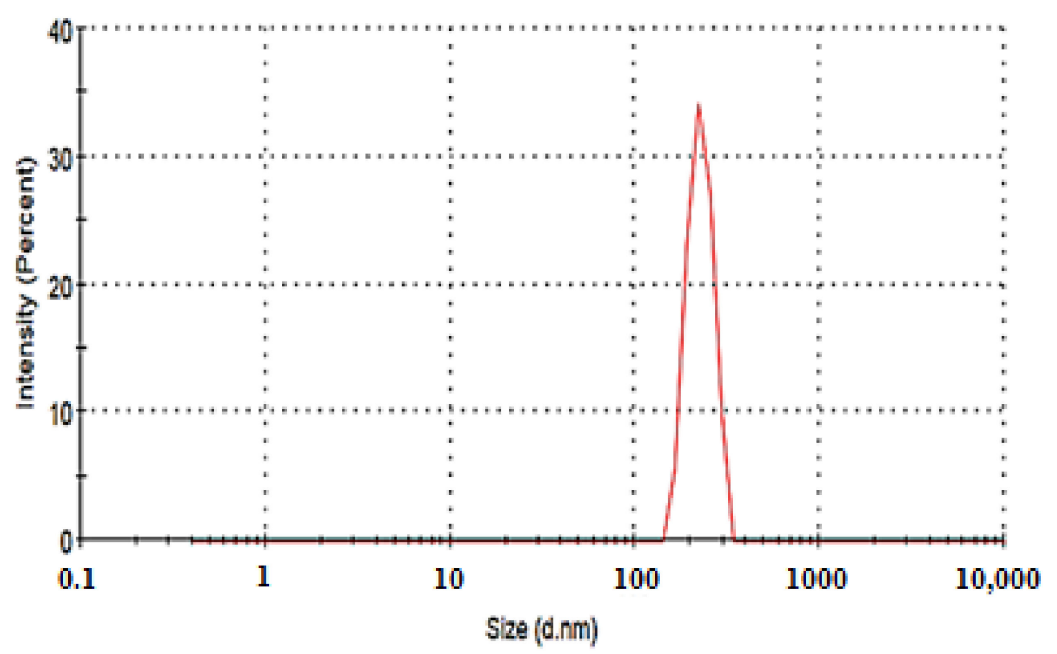

Figure 12. Particle size distribution curve of the optimized EGCG-loaded spanlastic formula (F4). Abbreviation: EGCG, epigallocatechin gallate.

High positive or negative zeta potential denotes the development of a stable colloidal dispersion because of the repulsion between the nanovesicles that permits the formation of a stable and non-agglomerated suspension [61]. The optimized EGCG-loaded spanlastic formula (F4) has a high negative zeta potential value $(-41.23 \mathrm{mv})$ indicating the stability of the selected spanlastic formula. The reason for the negative zeta potential value of nanovesicles, containing non-ionic surfactants, was discussed by some researchers on 
the basis of the adsorption of counterions at the vesicular surface [62], the orientation of non-ionic surfactant hydroxyl groups with respect to the aqueous medium [63], and the ionization of free groups present on the surface of nanovesicles [64].

The high cellular uptake of the negatively charged nanocarriers, without any repulsion with the negatively-charged cell membrane, might be due to the non-specific adsorption and development of clusters of nanovesicles on the cellular membrane [65].

\subsection{Pharmacokinetic Study}

The pharmacokinetic study was conducted in order to monitor the effect of encapsulating EGCG into the spanlastic and niosomal carriers on its bioavailability. The absorption of EGCG was tracked in rats by plotting plasma concentrations against time, as shown in Figure 13. Table 9 demonstrates different pharmacokinetic parameters. The order of increasing the $t_{1 / 2}$ was arranged as follows: EGCG-loaded SNVs $>$ niosomal formula $>$ EGCG dispersion. These findings exhibited the sustained release of EGCG from the spanlastic vesicles. Consequently, EGCG-loaded SNVs could maintain a sustained drug concentration in the plasma, which resulted in a reduction in the frequency of administration and improving patient compliance. Cai et al. [13] reported that the controlled release of EGCG is a feasible method to increase its bioavailability because free EGCG has too short $t_{1 / 2}$ to exert efficient clinical effects. Moreover, Safwat et al. [42] concluded that the ability of nanoparticles to hold EGCG for a sustained period of time is a crucial asset to increase the circulation time of the drug in the blood and to deliver it effectively to remote areas.

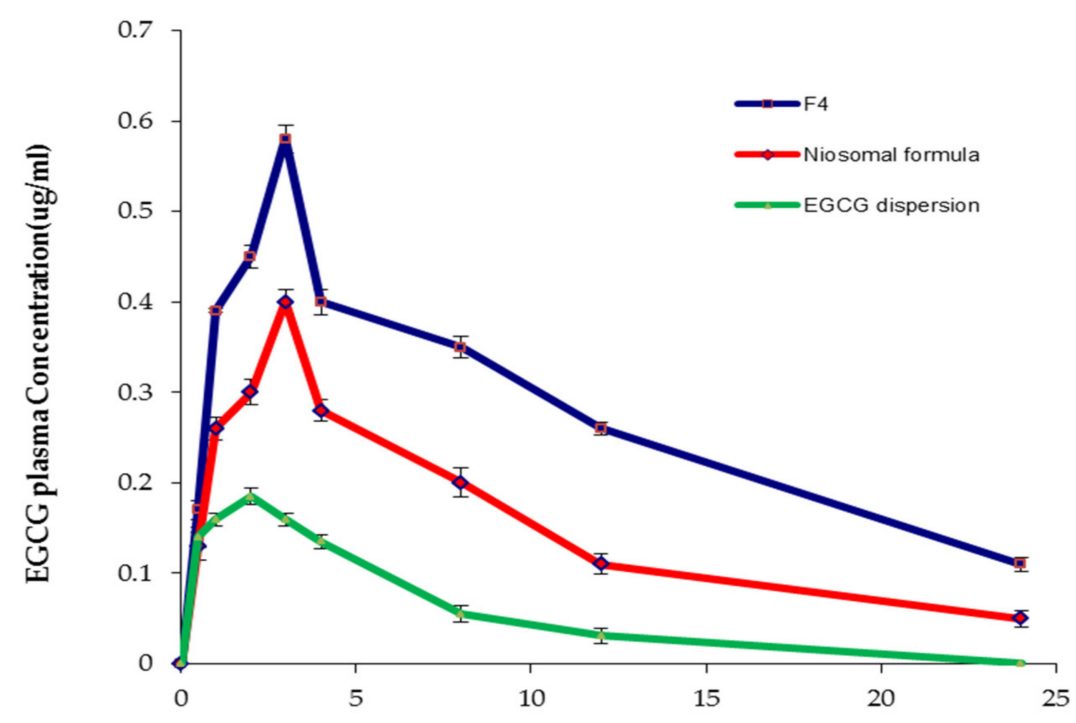

Time (h)

Figure 13. The plasma concentration-time curve of F4, the conventional niosomes, and EGCG dispersion. Abbreviation: EGCG, epigallocatechin gallate.

Table 9. Pharmacokinetic parameters of EGCG-loaded formulations.

\begin{tabular}{cccc}
\hline Parameter & EGCG-Loaded SNVs & EGCG-Loaded Niosomes & EGCG Dispersion \\
\hline $\mathrm{C}_{\max }\left(\mu \mathrm{g} \mathrm{ml}^{-1}\right)$ & $0.58 \pm 0.032$ & $0.40 \pm 0.025$ & $0.19 \pm 0.007$ \\
$\mathrm{AUC}_{0-24}\left(\mu \mathrm{g} \mathrm{ml}^{-1}\right)$ & $6.54 \pm 0.16$ & $4.24 \pm 0.12$ & $2.34 \pm 0.03$ \\
$\mathrm{t}_{1 / 2}(\mathrm{~h})$ & $17.43 \pm 0.34$ & $11.42 \pm 0.21$ & $3.7 \pm 0.11$ \\
$\mathrm{~F}_{\mathrm{re}} \%$ & 279.48 & 181.20 & $---{ }^{---}$ \\
\hline
\end{tabular}

Abbreviation: $\mathrm{C}_{\max }$ : maximum blood concentration; $\mathrm{AUC}$ : area under the EGCG concentration-time curve; $\mathrm{t}_{1 / 2}$ elimination half-life; $\mathrm{F}_{\mathrm{re}} \%$, \% relative bioavailability.

Additionally, the results showed that EGCG-loaded SNVs exhibited significantly higher $C_{\max }$ than the corresponding niosomal formula $(p<0.05)$ and the free EGCG 
dispersion $(p<0.001)$. The higher $C_{\max }$ of EGCG that attained post-administration of EGCG-loaded spanlastic formulation investigated the enhancement of EGCG absorption by loading into the SNVs.

The improvement in the oral bioavailability was also investigated from the results of the area under the curve $\left(\mathrm{AUC}_{0-24}\right)$ that showed a significant increase in the case of the optimized EGCG-loaded spanlastic formula than the conventional niosomal formula $(p<0.01)$ and EGCG dispersion $(p<0.001)$. Besides, the $\%$ relative bioavailability $\left(\mathrm{F}_{\mathrm{re}} \%\right)$ of EGCG-loaded spanlastics, in relation to free drug dispersion, was significantly $(p<0.01)$ higher than that of the corresponding niosomes.

The pharmacokinetic test, thus, supported the ex vivo intestinal permeation study of increasing permeability and then bioavailability of EGCG-loaded SNVs. Improved absorption and higher plasma concentration of EGCG after administering spanlastic and niosomal formulations may be attributed to improving the permeation of EGCG due to the presence of the basic nanocarrier constituents (surfactants) that act as permeation enhancers [66]. Moreover, entrapping EGCG into nanocarriers increases its stability by protection from enzymatic degradation [13]. These outcomes are in close agreement with Dube et al. [67], who reported that the mechanism of improving the absorption of catechins is the enhanced stabilization of drugs after encapsulation within nanoparticles. Additionally, Hu et al. [68] reported that a self-double-emulsifying solid formulation could improve the oral bioavailability and $C_{\max }$ of EGCG due to the protection of EGCG from the enzymatic degradation and alkaline/neutral conditions via encapsulation of drug and sustained drug release.

Additionally, the higher bioavailability of EGCG-loaded spanlastics could be explained on the basis of higher deformability and elasticity of SNVs due to the presence of EAs that increase the fluidity of the vesicular membrane and permit their passage through narrow pores of the biological membranes [23]. Accordingly, nanospanlastics not only improve the stability of EGCG, but also increase the membrane permeability due to their deformability.

\section{Materials and Methods}

\subsection{Materials}

EGCG, PEG-40 hydrogenated castor oil (Cremophor RH 40), polyoxyethylene (23) lauryl ether (Brij 35), and methanol (HPLC grade) were purchased from Sigma Chemical Co. (St. Louis, MI, USA). Sorbitan monostearate (Span 60), Sorbitan monooleate (Span 80), and cholesterol (CHOL) were obtained from Oxford Lab Chemicals (Mumbai, India). Potassium dihydrogen phosphate and potassium monohydrogen phosphate were provided from Alpha Chemica (Mumbai, India). Polyoxyethylene (20) sorbitan monooleate (Tween 80), absolute ethyl alcohol, and polyoxyethylene (20) sorbitan monostearate (Tween 60) were obtained from El-Nasr Chemical Co. Company (Cairo, Egypt). Spectra/Pore ${ }^{\circledR}$ dialysis membranes (Molecular weight cut-off 12,000-14,000 Dalton) were obtained from Spectrum Laboratories Inc. (Rancho Dominguez, CA, USA). All other chemicals, solvents, and reagents were of analytical grade.

\subsection{Methods}

3.2.1. HPLC Analysis of EGCG Using a Newly Developed and Validated RP-HPLC Method of Instrumentation

Dionex UltiMate 3000RS HPLC system (Thermo ScientificTM, DionexTM, Sunnyvale, CA, USA). It comprised of an LPG-3400RS quaternary pump, a WPS-3000RS autosampler, an Inertsil reversed-phase C18 $(150 \times 4.6 \mathrm{~mm} \times 5 \mu \mathrm{m})$ column, a TCC-3000RS column thermostat, and a DAD-3000RS diode array detector. Data collection and processing were performed by Chromeleon 7 software.

\section{Chromatographic Parameters}

A new simple and robust RP-HPLC method was developed and validated for EGCG quantification. The mobile phase used was $0.05 \mathrm{mM}$ potassium dihydrogen phosphate buffer ( $\mathrm{pH} 3$ ) and methanol at a 75:25 volume ratio. The injection volume was $20 \mu \mathrm{L}$ and 
the elution was performed at a flow rate of $1 \mathrm{~mL} / \mathrm{min}$. All assays were performed at room temperature. The detection wavelength of EGCG was $280 \mathrm{~nm}$.

Preparation of Standard and Calibration Solutions of EGCG

EGCG $(50 \mathrm{mg})$ was dissolved in $50 \mathrm{~mL}$ methanol with the aid of bath sonication (ultrasonic bath, Elmasonic E $30 \mathrm{H}$, Elma, Schmidbauer GmbH, Singen, Germany) for preparing the standard stock solution. The EGCG standard working solutions, in the concentration range of $100-1000 \mathrm{ng} / \mathrm{mL}$, have been prepared by diluting the stock standard solution with the mobile phase [69]. For each drug concentration level, triplicate injections of $20 \mu \mathrm{L}$ were chromatographed under the conditions described above. To avoid photodecomposition, the stock and working standard solutions of EGCG were kept away from light.

\section{Assay Validation}

The developed and optimized HPLC method was validated as guided in the ICH Q2B guidelines [70]. The following parameters of the current technique were validated: linearity, accuracy, precision, selectivity, the limit of detection (LOD), and the limit of quantification (LOQ), system suitability, and robustness [71]. In addition, the stability of ECGC in the selected mobile phase was also evaluated by keeping the standard solutions of EGCG away from the light, in tightly capped containers, on a laboratory bench, and in the refrigerator [72].

\subsubsection{Preliminary Screening Studies}

The preliminary screening studies were divided into two sections. The first section was conducted in order to choose the proper conditions (rotation speed and the sonication time) for the fabrication of EGCG-loaded spanlastics. EGCG-loaded spanlastic nanovesicles (SNVs) have been prepared using Span 60 as the non-ionic surfactant and Tween 80 as the EA at a 3:2 ratio at different rotation speeds (500 and $1000 \mathrm{rpm}$ ) for different times of sonication ( 3 and $5 \mathrm{~min}$ ), Table S1. The second section of the preliminary test aimed to choose the most suitable levels of different variables for the preparation of EGCG-loaded nanospanlastics. EGCG-loaded SNVs were formulated using three types of non-ionic surfactants (Span 60, Span 80, and Cremophor RH 40), three types of EAs (Tween 80, Tween 60, and Brij 35) at three ratios of non-ionic surfactant to EA (1:1, 3:2 and 4:1) (Table S2). The prepared spanlastic formulations were evaluated with respect to their $\mathrm{EE} \%$ that denotes the integrity of the vesicular membrane and the absence of drug leakage.

\subsubsection{Preparation of EGCG-Loaded Spanlastics}

Table 1 investigates the composition of different EGCG-loaded spanlastics. All excipients used for fabricating nanospanlastic formulations of EGCG are approved by the FDA [73] and generally recognized as safe (GRAS) [74]. A number of non-ionic SAAs were selected to be used as the membrane-forming lipids and EAs because they have considerable advantages over other classes of surfactants with respect to safety, compatibility, and stability [20]. EGCG-loaded spanlastics were fabricated by the ethanol injection method [75] due to its simple and reproducible technique [23,76]. EGCG and the non-ionic surfactants were dissolved carefully into $3 \mathrm{~mL}$ of absolute ethanol. The ethanolic solution was injected carefully into a preheated aqueous medium $\left(60^{\circ} \mathrm{C}\right)$ containing the EA and stirred continuously on a magnetic stirrer (Jenway 1000, Jenway, UK) till the formation of milky spanlastic dispersion $(10 \mathrm{~mL})$. For complete evaporation of any remaining alcohol, the spanlastic dispersion was stirred for another 1h. The EGCG-loaded spanlastic dispersions were then subjected to bath sonication (water-bath ultrasonicator, Elmasonic E $30 \mathrm{H}$, Elma, Schmidbauer $\mathrm{GmbH}$, Singen, Germany). The spanlastic formulations were then stored at $4{ }^{\circ} \mathrm{C}$ overnight for complete maturation of SNVs to be used for further studies. 


\subsubsection{Experimental Design}

A $2^{3}$ factorial design was performed using Design-Expert software, v7.0.0 (Stat-Ease, Inc., Minneapolis, MN, USA) to optimize the EGCG-loaded nanospanlastics [77]. The independent variables were the ratio of non-ionic surfactant: EA (X1), type of non-ionic surfactant (X2) and type of EA (X3). Dependent variables were the entrapment efficiency $(\mathrm{EE} \%, \mathrm{Y} 1)$, the percentage of drug released after $12 \mathrm{~h}\left(\mathrm{Q}_{12 \mathrm{~h}}, \mathrm{Y} 2\right)$, and the particle size (PS, Y3). Each factor was screened at two levels; the lower level (-1) and the upper level $(+1)$, respectively.

The coefficient of determination $\left(R^{2}\right)$ and predicted/adjusted $R^{2}$ of the chosen model were calculated for exploring its goodness of fit to the experimental results. Additionally, the significance level of the studied terms was investigated by the analysis of variance (ANOVA) [78].

\subsubsection{In Vitro Characterization of EGCG-Loaded Nanospanlastics}

Determination of Drug Content and Entrapment Efficiency of EGCG-Loaded Nanospanlastics

The EE\% of EGCG-loaded SNVs was determined using ultracentrifugation according to the indirect method in order to separate the un-entrapped drug, and $1 \mathrm{~mL}$ of EGCG-loaded SNVs was centrifuged at 20,000 rpm at $4^{\circ} \mathrm{C}$ for $1 \mathrm{~h}$ (Cooling centrifuge, HermleLabortechnik $\mathrm{GmbH}$, Germany). The supernatant was passed carefully through a $0.20 \mu \mathrm{m}$ pore size nylon membrane filter (Nylon Acrodisc, Gelman Sciences Inc., Ann Arbor, MI, USA) and the concentration of free EGCG was measured by HPLC at $280 \mathrm{~nm}$. EE\% was calculated as follows [19]:

$\mathrm{EE}(\%)=($ Total amount of EGCG - Amount of free EGCG) $\times 100 /$ Total amount of EGCG

The total drug content of EGCG (unentrapped + entrapped) was estimated via disrupting the spanlastic dispersions $(1 \mathrm{~mL})$ using isopropyl alcohol $(100 \mathrm{~mL})$ [79]. The samples were filtered, diluted, and analyzed for drug content using HPLC at $280 \mathrm{~nm}$.

\section{In Vitro Release Study of EGCG-Loaded SNVs}

Prior to conducting the in vitro release test, the solubility of EGCG was estimated in phosphate-buffered saline $(\mathrm{pH}=7.4)$ using the shake-flask method [80] to ensure attaining sink conditions of the chosen dissolution medium.

The membrane diffusion technique was used for studying the in vitro release profile of EGCG-loaded nanospanlastics [79]. The semi-permeable cellulose membrane was prehydrated carefully using a phosphate-buffered saline solution $(\mathrm{pH}=7.4)$ at room temperature for $24 \mathrm{~h}$. The cellulose membrane was fixed, using a rubber band, at the end of a glass cylinder attached to the dissolution apparatus shaft (USP apparatus II, Erweka DT-720, Kreuzau, Germany). The receptor medium was $300 \mathrm{~mL}$ phosphate-buffered saline solution of $\mathrm{pH}=7.4$ [81]. The receptor medium was stirred at $50 \mathrm{rpm}$ and kept at $37 \pm 0.5^{\circ} \mathrm{C} .1 \mathrm{~mL}$ of the spanlastic formulations containing entrapped EGCG or the equivalent concentration of EGCG dispersion were introduced in the donor chamber over the cellulose membrane; $200 \mu \mathrm{L}$ aliquots were withdrawn at the predetermined time intervals and substituted by an equal volume of fresh buffer solution to preserve a constant volume of the receptor medium [77]. The withdrawn samples were filtered through a $0.20 \mu \mathrm{m}$ nylon membrane filter (Nylon Acrodisc, Gelman Sciences Inc., Ann Arbor, MI, USA), diluted and analyzed using HPLC at $280 \mathrm{~nm}$. Triplicate experiments were performed. The results are expressed as mean values $\pm S D$. The mechanism of the in vitro release of EGCG from the SNVs was determined by a kinetic study using different mathematical models [38].

Determination of the Particle Size (PS) and Polydispersity Index (PDI) of EGCG-Loaded SNVs

The colloidal characters of the EGCG-loaded nanospanlastic dispersions were investigated by determination of PS and PDI. The EGCG-loaded nanospanlastic formulations $(1 \mathrm{~mL})$ were diluted by deionized water $(100 \mathrm{~mL})$ in order to get a reasonable scattering 
intensity. Then, the vesicle size and PDI of EGCG-loaded SNVs were determined using a particle size analyzer (NICOMP 380 ZLS Zeta Potential/Particle Sizer, Santa Barbara, CA, USA) $[23,31]$. Different measurements were performed in triplicate and the mean of three runs were determined.

\subsubsection{Statistical Optimization of EGCG-Loaded SNVs}

The desirability values were used to select the optimized formula because they describe the closeness of the chosen responses to their optimal values [82]. The formulation that has the highest desirability value is selected as the optimized formula [23]. In the present study, the optimized formula was selected on the basis of maximum $\mathrm{EE} \%$, maximum $Q_{12 h}$, and minimum PS. Additionally, the optimized EGCG-loaded spanlastic formula was validated by calculating \% relative error via comparing the observed responses ( $Y 1$, $\mathrm{Y} 2$, and $\mathrm{Y} 3$ ) with their corresponding predicted values [23] as follows:

$\%$ Relative error $=($ predicted value-observed value $) \times 100 /$ predicted value

The optimized EGCG-loaded nanospanlastic formula was subjected to a comparative study with the corresponding niosomes and evaluated by additional characterization tests.

3.2.7. Comparative Study of the Optimized EGCG-Loaded Nanospanlastic Formula and the Conventional Niosome

Preparation of EGCG-Loaded Conventional Niosomes

The conventional EGCG-loaded niosomes were fabricated by the ethanol injection technique $[56,83]$ using the same previous steps described for preparing the spanlastic dispersion without the addition of EA and with the addition of CHOL to the non-ionic surfactant at a 1:2 molar ratio.

Evaluation of EE\%, Drug Content and the In Vitro Release Study

The EE\%, drug content, and the in vitro release of EGCG-loaded niosomes were studied as previously described in the in vitro characterization section of spanlastics.

\section{Ex Vivo Intestinal Permeation Study}

The ex vivo intestinal permeation test was performed using 200-220 g Male Wistar rats after approving the study protocol by the committee of ethics, the Faculty of Pharmacy, Kafrelsheikh University, Egypt (Approval number KFS-2020/02) and according to the ethical guidelines [84-86]. After sacrificing rats under anesthesia, the small intestines were carefully removed and rinsed thoroughly with saline solution $(0.9 \%)$ for removal of any mucosal or luminal contents $[87,88]$.

The ex vivo permeation profile of the optimized EGCG-loaded nanospanlastic formula was compared with the corresponding niosomal dispersion and the aqueous dispersion of EGCG. The receptor medium was $300 \mathrm{~mL}$ phosphate-buffered saline solution of $\mathrm{pH}=7.4$ [81] that stirred at $50 \mathrm{rpm}$ and kept at $37 \pm 0.5^{\circ} \mathrm{C}$. The excised intestinal tract was cut into sacs that were filled with the tested formulations $(1 \mathrm{~mL})$. The two sides of the intestinal sac were tied with a surgical thread and attached to the shafts of the dissolution apparatus [87,89]; $200 \mu \mathrm{L}$ sample was withdrawn at the predetermined time intervals $(1,2,3,4,8$, and $12 \mathrm{~h})$ and replaced by the same volume of fresh buffer. Samples were passed through a $0.20 \mu \mathrm{m}$ pore size nylon membrane filter (Nylon Acrodisc, Gelman Sciences Inc., Ann Arbor, Michigan, USA), diluted and analyzed using HPLC at $280 \mathrm{~nm}$. The experiment was conducted in triplicate and the \% EGCG permeated was described as the average \pm SD. The ex vivo permeation graphs were plotted by comparing $\%$ EGCG permeated with respect to time.

Different pharmacokinetic parameters were estimated such as the steady-state flux of EGCG $\left(\mathrm{J}_{\mathrm{ss}}\right)$, permeability coefficient $\left(\mathrm{K}_{\mathrm{p}}\right)$, and the enhancement ratio (ER) of both EGCGloaded spanlastic and niosomal formulations [77]. The mechanism of the ex vivo intestinal 
permeation of EGCG was determined by a kinetic study using different mathematical models [38].

\section{Measurement of Vesicle Deformability}

The deformability of the optimized nanospanlastic formula and the conventional niosomes was demonstrated by extrusion through a $100 \mathrm{~nm}$ nylon membrane filter for 5 $\mathrm{min}$ at 2.5 bar [90]. The vesicle size (before and after extrusion) of both the spanlastic and niosomal formulations was determined by a NICOMP 380 ZLS Zeta Potential/Particle Sizer (PSS Nicomp, Santa Barbara, CA, USA). The deformability index (DI) was calculated using the following equation:

$$
\mathrm{DI}=\mathrm{J}\left(\mathrm{r}_{\mathrm{v}} / \mathrm{r}_{\mathrm{p}}\right)^{2}
$$

where $\mathrm{J}$ is the amount of the extruded EGCG-loaded spanlastic/niosomal dispersion, $\mathrm{r}_{\mathrm{v}}$ : the vesicle size of the extruded EGCG-loaded spanlastic/niosomal formula and $r_{p}$ : the pore size of the nylon membrane filter.

The deformability, in terms of DI, was estimated by the extrusion method in order to compare the elasticity of the optimized EGCG-loaded SNVs with that of the conventional niosomal dispersion. This test could demonstrate the effect of EAs on the elasticity of spanlastic vesicles that permit them to pass through narrow pores of biological membranes. Besides, the vesicle size of both formulations was estimated before and after extrusion to investigate the effect of adding EAs on retaining the vesicular size after extrusion that prevents the rupture of the SNVs during passage through different membranes.

The Stability Test

Both the optimized EGCG-loaded spanlastic formula and the corresponding niosomal dispersion were kept at $4-8{ }^{\circ} \mathrm{C}$ in tightly closed glass vials for three months [40]. The EGCG-loaded spanlastic and the niosomal formulations were evaluated with regard to their $\mathrm{EE} \%$, drug content, $\mathrm{Q}_{12 \mathrm{~h}}$ \% drug permeated, and DI to investigate the influence of storage conditions on the stability of both formulations.

\subsubsection{Characterization of the Optimized EGCG-Loaded SNVs}

Scanning Electron Microscopy (SEM)

The optimized EGCG-loaded nanospanlastic formula $(1 \mathrm{~mL})$ was suitably diluted using deionized water $(100 \mathrm{~mL})$ and affixed carefully onto the SEM sample aluminum stub with a sticking carbon tape. Afterward, the EGCG spanlastic dispersion was subjected to vacuum drying overnight followed by coating with gold film. After coating, the EGCGloaded spanlastic vesicles were examined by scanning electron microscope (JSM-6360, JEOL, Tokyo, Japan) [91].

\section{Determination of Particle Size and Zeta Potential}

The optimized EGCG-loaded nanospanlastic dispersion $(1 \mathrm{~mL})$ was suitably diluted using deionized water $(100 \mathrm{~mL})$. The particle size and zeta potential of SNVs have been estimated, on the basis of a light scattering technique, using a particle size analyzer (NICOMP 380 ZLS Zeta Potential/Particle Sizer, Santa Barbara, CA, USA) via detection of the electrophoretic mobility of EGCG-loaded SNVs within the electric field [31,92].

\subsubsection{Pharmacokinetic Study}

Male Wistar rats (200-220 g) were used in the pharmacokinetic study to compare the in vivo performance of EGCG-loaded SNVs with that of the corresponding niosomes and the free EGCG dispersion. The rats were obtained from the National Research Center (Dokki, Giza, Egypt) and kept in sawdust-bedded cages within a pathogen-free facility. The animal rooms were maintained at $50 \%$ relative humidity, at a controlled temperature of $25 \pm 2{ }^{\circ} \mathrm{C}$, and under $12 \mathrm{~h}$-light, $12 \mathrm{~h}$-dark cycles. Adaptation of the tested rats was conducted, under standard conditions, within the animal house for 14 days before performing the experiment. The experimental protocol and animals used were approved by 
the Committee of Ethics, Faculty of Pharmacy, Kafrelsheikh University, Egypt (Approval number KFS-2020/08). The methodology of the pharmacokinetic study was in accordance with the ethical guidelines [84-86].

Rats were randomly divided into three groups (6 rats each). Before performing the experiment, the rats were fasted overnight with free access to water. Group A administered EGCG-loaded spanlastic dispersion, group B administered the corresponding niosomal dispersion, and group C administered EGCG dispersion. Each dispersion, equivalent to $50 \mathrm{mg} / \mathrm{kg}$ of EGCG, [93,94], was administered orally by a gavage tube. Blood samples were withdrawn, from the tail vein, into heparin-containing tubes, immediately prior to dosing (zero time) and then after administration of EGCG formulations at 0.5, 1, 2, 3, 4, 8, 12, and $24 \mathrm{~h}$. Different blood samples were centrifuged at $15,000 \mathrm{rpm}$ for $10 \mathrm{~min}$ and $100 \mu \mathrm{L}$ plasma samples were then collected, vortex-mixed for $1 \mathrm{~min}$, and centrifuged at $5000 \mathrm{rpm}$ for $5 \mathrm{~min}$. The drug concentration was estimated using an HPLC assay at $280 \mathrm{~nm}$ and different pharmacokinetic parameters were determined using the plasma concentration-time curve, such as the maximum plasma concentration $\left(\mathrm{C}_{\max }\right)$, the area under the curve $\left(\mathrm{AUC}_{0-24}\right)$, and the half-life $\left(\mathrm{t}_{1 / 2}\right)$. The $\%$ relative bioavailability $\left(\mathrm{F}_{\text {rel }}\right)$ of the niosomal and spanlastic dispersions was calculated in relation to free EGCG dispersion [95].

\subsubsection{Statistical Analysis}

SPSS-11 software (SPSS. Inc., Chicago. IL, USA) was used for the statistical analysis of the results. The data, obtained from the $2^{3}$ factorial design, were analyzed using ANOVA by Design-Expert software (Stat-Ease, Inc., Minneapolis, Minnesota, USA) to investigate the effect of the selected variables on EE\%, $\mathrm{Q}_{12 \mathrm{~h}}$, and PS of EGCG-loaded SNVs. $p$-values lower than 0.05 denote statistically significant variations between the tested terms.

\section{Conclusions}

The current study demonstrated the preparation of EGCG-loaded spanlastics as an effective and flexible nanocarrier for improving the bioavailability of EGCG. Eight EGCGloaded nanospanlastics were effectively fabricated using the ethanol injection method according to $2^{3}$ factorial design. The optimized spanlastic formulation (F4) was chosen according to the desirability criteria on the basis of maximizing both $E E \%$ and $\mathrm{Q}_{12 \mathrm{~h}}$ and minimizing PS. Using the simple and validated RP-HPLC method helped in rapid and robust determination of ECGC. Upon comparing with the conventional niosomes, F4 demonstrated more prolonged drug release, with higher $\mathrm{EE} \%$, deformability, stability, and bioavailability. In brief, these outcomes exhibited that EGCG-loaded spanlastics are a promising and flexible nanocarrier that could overcome the shortcomings of the limited permeation and poor stability of EGCG.

Supplementary Materials: The following are available online at https:/ /www.mdpi.com/1424-824 7/14/1/68/s1, Table S1: The prescreening study for the formulation of EGCG-loaded SNVs using different rotation, Table S2: Prescreening study for selecting the most suitable levels of different independent variables required for preparing EGCG-loaded SNVs.

Author Contributions: Conceptualization, E.A.M.; methodology, E.A.M., M.Y., M.M.E., and A.G.A.E.; software, E.A.M., and A.G.A.E.; validation, E.A.M., D.A.H., and M.M.E.; formal analysis, E.A.M., M.Y. and A.G.A.E.; investigation, E.A.M. and M.Y.; resources, E.A.M., M.M.E., and D.A.H.; data curation, E.A.M., M.Y. and D.A.H.; writing—original draft preparation, E.A.M. and M.M.E.; writing—review and editing, E.A.M. and M.Y.; visualization, E.A.M., M.M.E. and A.G.A.E.; supervision, M.Y., and A.G.A.E. All authors have read and agreed to the published version of the manuscript.

Funding: This research received no external funding.

Institutional Review Board Statement: The experimental protocol and animals used were ap-proved by the Committee of Ethics, Faculty of Pharmacy, Kafrelsheikh University, Egypt (Ap-proval number KFS-2020/08).

Informed Consent Statement: Not applicable. 
Data Availability Statement: The data presented in the current study are available from the corresponding author upon reasonable request.

Conflicts of Interest: The authors declare no conflict of interest.

\section{References}

1. Rashidinejad, A.; Birch, E.J.; Sun-Waterhouse, D.; Everett, D.W. Delivery of green tea catechin and epigallocatechin gallate in liposomes incorporated into low-fat hard cheese. Food Chem. 2014, 156, 176-183. [CrossRef] [PubMed]

2. Dai, W.; Ruan, C.; Zhang, Y.; Wang, J.; Han, J.; Shao, Z.; Sun, Y.; Liang, J. Bioavailability enhancement of EGCG by structural modification and nano-delivery: A review. J. Funct. Foods 2020, 65, 103732. [CrossRef]

3. Ramadan, G.; Nadia, M.; Abd El-Ghffar, E.A. Modulatory effects of black v. green tea aqueous extract on hyperglycaemia, hyperlipidaemia and liver dysfunction in diabetic and obese rat models. Br. J. Nutr. 2009, 102, 1611-1619. [CrossRef] [PubMed]

4. Al-Sayed, E.; Abdel-Daim, M.M. Analgesic and anti-inflammatory activities of epicatechin gallate from Bauhinia hookeri. Drug Dev. Res. 2018, 79, 157-164. [CrossRef]

5. Pandit, A.P.; Joshi, S.R.; Dalal, P.S.; Patole, V.C. Curcumin as a permeability enhancer enhanced the antihyperlipidemic activity of dietary green tea extract. BMC Complement. Altern. Med. 2019, 19, 1-10. [CrossRef]

6. Fujiki, H.; Suganuma, M. Green tea: An effective synergist with anticancer drugs for tertiary cancer prevention. Cancer Lett. 2012, 324, 119-125. [CrossRef]

7. Grassi, D.; Desideri, G.; Di Giosia, P.; De Feo, M.; Fellini, E.; Cheli, P.; Ferri, L.; Ferri, C. Tea, flavonoids, and cardiovascular health Endothelial protection. Am. J. Clin. Nutr. 2013, 98, 1660S-1666S. [CrossRef]

8. Yu, Y.; Deng, Y.; Lu, B.-M.; Liu, Y.-X.; Li, J.; Bao, J.-K. Green tea catechins: A fresh flavor to anticancer therapy. Apoptosis 2014, 19, 1-18. [CrossRef]

9. Gu, J.-W.; Makey, K.L.; Tucker, K.B.; Chinchar, E.; Mao, X.; Pei, I.; Thomas, E.Y.; Miele, L. EGCG, a major green tea catechin suppresses breast tumor angiogenesis and growth via inhibiting the activation of HIF-1 $\alpha$ and NFKB, and VEGF expression. Vasc. Cell 2013, 5, 1-10. [CrossRef]

10. Crespy, V.; Williamson, G. A review of the health effects of green tea catechins in in vivo animal models. J. Nutr. 2004, 134, 3431S-3440S. [CrossRef]

11. Prasanth, M.I.; Sivamaruthi, B.S.; Chaiyasut, C.; Tencomnao, T. A review of the role of green tea (Camellia sinensis) in antiphotoaging, stress resistance, neuroprotection, and autophagy. Nutrients 2019, 11, 474. [CrossRef] [PubMed]

12. Baba, S.; Osakabe, N.; Natsume, M.; Muto, Y.; Takizawa, T.; Terao, J. In vivo comparison of the bioavailability of (+)-catechin,(-)epicatechin and their mixture in orally administered rats. J. Nutr. 2001, 131, 2885-2891. [CrossRef] [PubMed]

13. Cai, Z.-Y.; Li, X.-M.; Liang, J.-P.; Xiang, L.-P.; Wang, K.-R.; Shi, Y.-L.; Yang, R.; Shi, M.; Ye, J.-H.; Lu, J.-L. Bioavailability of tea catechins and its improvement. Molecules 2018, 23, 2346. [CrossRef] [PubMed]

14. Song, Q.; Li, D.; Zhou, Y.; Yang, J.; Yang, W.; Zhou, G.; Wen, J. Enhanced uptake and transport of (+)-catechin and (-)epigallocatechin gallate in niosomal formulation by human intestinal Caco-2 cells. Int. J. Nanomed. 2014, 9, 2157. [CrossRef] [PubMed]

15. Dube, A.; Ng, K.; Nicolazzo, J.A.; Larson, I. Effective use of reducing agents and nanoparticle encapsulation in stabilizing catechins in alkaline solution. Food Chem. 2010, 122, 662-667. [CrossRef]

16. Mahale, N.; Thakkar, P.; Mali, R.; Walunj, D.; Chaudhari, S. Niosomes: Novel sustained release nonionic stable vesicular systems-An overview. Adv. Colloid Interface Sci. 2012, 183, 46-54. [CrossRef]

17. Zou, L.-Q.; Peng, S.-F.; Liu, W.; Gan, L.; Liu, W.-1.; Liang, R.-H.; Liu, C.-M.; Niu, J.; Cao, Y.-1.; Liu, Z. Improved in vitro digestion stability of (-)-epigallocatechin gallate through nanoliposome encapsulation. Food Res. Int. 2014, 64, 492-499. [CrossRef]

18. Badria, F.; Mazyed, E. Formulation of Nanospanlastics as a Promising Approach for Improving the Topical Delivery of a Natural Leukotriene Inhibitor (3- Acetyl-11-Keto- $\beta$-Boswellic Acid): Statistical Optimization, in vitro Characterization, and ex vivo Permeation Study. Drug Des. Dev. Ther. 2020, 14, 3697. [CrossRef]

19. Al-Mahallawi, A.M.; Khowessah, O.M.; Shoukri, R.A. Enhanced non invasive trans-tympanic delivery of ciprofloxacin through encapsulation into nano-spanlastic vesicles: Fabrication, in-vitro characterization, and comparative ex-vivo permeation studies. Int. J. Pharm. 2017, 522, 157-164. [CrossRef]

20. Kakkar, S.; Kaur, I.P. Spanlastics-A novel nanovesicular carrier system for ocular delivery. Int. J. Pharm. 2011, 413, 202-210. [CrossRef]

21. Zheng, W.-S.; Fang, X.-Q.; Wang, L.-1.; Zhang, Y.-J. Preparation and quality assessment of itraconazole transfersomes. Int. J. Pharm. 2012, 436, 291-298. [CrossRef] [PubMed]

22. Kamboj, S.; Saini, V.; Magon, N.; Bala, S.; Jhawat, V. Vesicular drug delivery systems: A novel approach for drug targeting. Brain 2013, 1, 11 .

23. Badria, F.; Fayed, H.A.; Ibraheem, A.K.; Mazyed, E.A. Formulation of Sodium Valproate Nanospanlastics as a Promising Approach for Drug Repurposing in the Treatment of Androgenic Alopecia. Pharmaceutics 2020, 12, 866. [CrossRef] [PubMed]

24. McCalley, D.V. Selection of suitable stationary phases and optimum conditions for their application in the separation of basic compounds by reversed-phase HPLC. J. Sep. Sci. 2003, 26, 187-200. [CrossRef]

25. Hopkins, T. The role of methanol and acetonitrile as organic modifiers in reversed-phase liquid chromatography. Chromatogr. Today 2019, 26, 24-26. 
26. European Agency for the Evaluation of Medical Products. ICH Topic Q 2B Note for Guidance on Validation of Analytical Procedures: Methodology; European Agency for the Evaluation of Medical Products: Amsterdam, The Netherlands, 1996.

27. Li, D.; Martini, N.; Wu, Z.; Wen, J. Development of an isocratic HPLC method for catechin quantification and its application to formulation studies. Fitoterapia 2012, 83, 1267-1274. [CrossRef]

28. Yassin, G.E.; Amer, R.I.; Fayez, A.M. Carbamazepine loaded vesicular structures for enhanced brain targeting via intranasal route: Optimization, in vitro evaluation, and in vivo study. Int. J. Appl. Pharm. 2019, 11, 264-274. [CrossRef]

29. Ngan, C.L.; Basri, M.; Lye, F.F.; Masoumi, H.R.F.; Tripathy, M.; Karjiban, R.A.; Abdul-Malek, E. Comparison of process parameter optimization using different designs in nanoemulsion-based formulation for transdermal delivery of fullerene. Int. J. Nanomed. 2014, 9, 4375. [CrossRef]

30. Li, P.-H.; Chiang, B.-H. Process optimization and stability of D-limonene-in-water nanoemulsions prepared by ultrasonic emulsification using response surface methodology. Ultrason. Sonochem. 2012, 19, 192-197. [CrossRef]

31. Fahmy, A.M.; El-Setouhy, D.A.; Ibrahim, A.B.; Habib, B.A.; Tayel, S.A.; Bayoumi, N.A. Penetration enhancer-containing spanlastics (PECSs) for transdermal delivery of haloperidol: In vitro characterization, ex vivo permeation and in vivo biodistribution studies. Drug Deliv. 2018, 25, 12-22. [CrossRef]

32. Pandit, J.; Garg, M.; Jain, N.K. Miconazole nitrate bearing ultraflexible liposomes for the treatment of fungal infection. J. Liposome Res. 2014, 24, 163-169. [CrossRef] [PubMed]

33. Abdelbary, G.; El-Gendy, N. Niosome-encapsulated gentamicin for ophthalmic controlled delivery. AAPS Pharmscitech 2008, 9 , 740-747. [CrossRef] [PubMed]

34. Araujo, J.; Gonzalez-Mira, E.; Egea, M.; Garcia, M.; Souto, E. Optimization and physicochemical characterization of a triamcinolone acetonide-loaded NLC for ocular antiangiogenic applications. Int. J. Pharm. 2010, 393, 168-176. [CrossRef] [PubMed]

35. Aziz, D.E.; Abdelbary, A.A.; Elassasy, A.I. Implementing Central Composite Design for Developing Transdermal DiacereinLoaded Niosomes: Ex vivo Permeation and In vivo Deposition. Curr. Drug Deliv. 2018, 15, 1330-1342. [CrossRef] [PubMed]

36. Fang, J.-Y.; Yu, S.-Y.; Wu, P.-C.; Huang, Y.-B.; Tsai, Y.-H. In vitro skin permeation of estradiol from various proniosome formulations. Int. J. Pharm. 2001, 215, 91-99. [CrossRef]

37. Nematollahi, M.H.; Pardakhty, A.; Torkzadeh-Mahanai, M.; Mehrabani, M.; Asadikaram, G. Changes in physical and chemical properties of niosome membrane induced by cholesterol: A promising approach for niosome bilayer intervention. RSC Adv. 2017, 7, 49463-49472. [CrossRef]

38. Dharashivkar, S.; Sahasrabuddhe, S.; Saoji, A. Silver sulfadiazine niosomes: A novel sustained release once a day formulation for burn treatment. Int. J. Pharm. Pharm. Sci. 2014, 6, 611-616.

39. Ruckmani, K.; Sankar, V. Formulation and optimization of zidovudine niosomes. AAPS Pharmscitech 2010, 11, 1119-1127. [CrossRef]

40. Sambhakar, S.; Paliwal, S.; Sharma, S.; Singh, B. Formulation of risperidone loaded proniosomes for effective transdermal delivery: An in-vitro and in-vivo study. Bull. Fac. Pharm. Cairo Univ. 2017, 55, 239-247. [CrossRef]

41. Mazyed, E.A.; Abdelaziz, A.E. Fabrication of Transgelosomes for Enhancing the Ocular Delivery of Acetazolamide: Statistical Optimization, In Vitro Characterization, and In Vivo Study. Pharmaceutics 2020, 12, 465. [CrossRef]

42. Safwat, M.A.; Kandil, B.A.; Elblbesy, M.A.; Soliman, G.M.; Eleraky, N.E. Epigallocatechin-3-Gallate-Loaded Gold Nanoparticles: Preparation and Evaluation of Anticancer Efficacy in Ehrlich Tumor-Bearing Mice. Pharmaceuticals 2020, 13, 254. [CrossRef] [PubMed]

43. El Zaafarany, G.M.; Awad, G.A.; Holayel, S.M.; Mortada, N.D. Role of edge activators and surface charge in developing ultradeformable vesicles with enhanced skin delivery. Int. J. Pharm. 2010, 397, 164-172. [CrossRef] [PubMed]

44. Ramadan, A.A.; Eladawy, S.A.; El-Enin, A.S.M.A.; Hussein, Z.M. Development and investigation of timolol maleate niosomal formulations for the treatment of glaucoma. J. Pharm. Investig. 2020, 50, 59-70. [CrossRef]

45. Khoee, S.; Yaghoobian, M. Niosomes: A novel approach in modern drug delivery systems. In Nanostructures for Drug Delivery; Elsevier: Amsterdam, The Netherlands, 2017; pp. 207-237.

46. Taymouri, S.; Varshosaz, J. Effect of different types of surfactants on the physical properties and stability of carvedilol nanoniosomes. Adv. Biomed. Res. 2016, 5. [CrossRef]

47. Baker, R. Controlled release: Mechanisms and rates. Control. Release Biol. Act. Agents 1974, 15, 15-71.

48. Higuchi, T. Rate of release of medicaments from ointment bases containing drugs in suspension. J. Pharm. Sci. 1961, 50, 874-875. [CrossRef]

49. Dora, C.P.; Singh, S.K.; Kumar, S.; Datusalia, A.K.; Deep, A. Development and characterization of nanoparticles of glibenclamide by solvent displacement method. Acta Pol. Pharm. 2010, 67, 283-290.

50. Akbari, V.; Abedi, D.; Pardakhty, A.; Sadeghi-Aliabadi, H. Release studies on ciprofloxacin loaded non-ionic surfactant vesicles. Avicenna J. Med. Biotechnol. 2015, 7, 69. [PubMed]

51. Yoshioka, T.; Sternberg, B.; Florence, A.T. Preparation and properties of vesicles (niosomes) of sorbitan monoesters (Span 20, 40, 60 and 80) and a sorbitan triester (Span 85). Int. J. Pharm. 1994, 105, 1-6. [CrossRef]

52. ElMeshad, A.N.; Mohsen, A.M. Enhanced corneal permeation and antimycotic activity of itraconazole against Candida albicans via a novel nanosystem vesicle. Drug Deliv. 2016, 23, 2115-2123. [CrossRef] [PubMed]

53. Basalious, E.B.; Shawky, N.; Badr-Eldin, S.M. SNEDDS containing bioenhancers for improvement of dissolution and oral absorption of lacidipine. I: Development and optimization. Int. J. Pharm. 2010, 391, 203-211. [CrossRef] [PubMed] 
54. John, B. Application of desirability function for optimizing the performance characteristics of carbonitrided bushes. Int. J. Ind. Eng. Comput. 2013, 4, 305-314. [CrossRef]

55. Salama, H.A.; Mahmoud, A.A.; Kamel, A.O.; Abdel Hady, M.; Awad, G.A. Brain delivery of olanzapine by intranasal administration of transfersomal vesicles. J. Liposome Res. 2012, 22, 336-345. [CrossRef] [PubMed]

56. Manosroi, A.; Jantrawut, P.; Manosroi, J. Anti-inflammatory activity of gel containing novel elastic niosomes entrapped with diclofenac diethylammonium. Int. J. Pharm. 2008, 360, 156-163. [CrossRef] [PubMed]

57. Leonyza, A.; Surini, S. Optimization of sodium deoxycholate-based transfersomes for percutaneous delivery of peptides and proteins. Int. J. Appl. Pharm. 2019, 11, 329-332. [CrossRef]

58. Garg, V.; Singh, H.; Bhatia, A.; Raza, K.; Singh, S.K.; Singh, B.; Beg, S. Systematic development of transethosomal gel system of piroxicam: Formulation optimization, in vitro evaluation, and ex vivo assessment. AAPS Pharmscitech 2017, 18, 58-71. [CrossRef]

59. Das, M.K.; Palei, N.N. Sorbitan ester niosomes for topical delivery of rofecoxib. Indian J. Exp. Biol. 2011, 49, 438-445.

60. Mazyed, E.A.; Zakaria, S. Enhancement of dissolution characteristics of clopidogrel bisulphate by proniosomes. Int. J. Appl. Pharm. 2019, 11, 77-85. [CrossRef]

61. Sengodan, T.; Sunil, B.; Vaishali, R.; Chandra, R.J.; Nagar, S.; Nagar, O. Formulation and evaluation of maltodextrin based proniosomes loaded with indomethacin. Int. J. Pharmtech Res. 2009, 1, 517-523.

62. Junyaprasert, V.B.; Teeranachaideekul, V.; Supaperm, T. Effect of charged and non-ionic membrane additives on physicochemical properties and stability of niosomes. AAPS Pharmscitech 2008, 9, 851. [CrossRef]

63. Caracciolo, G.; Pozzi, D.; Caminiti, R.; Marianecci, C.; Moglioni, S.; Carafa, M.; Amenitsch, H. Effect of hydration on the structure of solid-supported Niosomal membranes investigated by in situ energy dispersive X-ray diffraction. Chem. Phys. Lett. 2008, 462, 307-312. [CrossRef]

64. Pawar, H.A.; Attarde, V.B.; Parag Subhash, G. Optimization of Bifonazole-Loaded Nisomal Formulation Using Plackett-Burman Design and 23 Factorial Design. Open Pharm. Sci. J. 2016, 3, 31-48. [CrossRef]

65. Uchechi, O.; Ogbonna, J.D.; Attama, A.A. Nanoparticles for dermal and transdermal drug delivery. Appl. Nanotechnol. Drug Deliv. 2014, 4, 193-227.

66. Arzani, G.; Haeri, A.; Daeihamed, M.; Bakhtiari-Kaboutaraki, H.; Dadashzadeh, S. Niosomal carriers enhance oral bioavailability of carvedilol: Effects of bile salt-enriched vesicles and carrier surface charge. Int. J. Nanomed. 2015, $10,4797$.

67. Dube, A.; Nicolazzo, J.A.; Larson, I. Chitosan nanoparticles enhance the intestinal absorption of the green tea catechins (+)-catechin and (-)-epigallocatechin gallate. Eur. J. Pharm. Sci. 2010, 41, 219-225. [CrossRef]

68. Hu, C.; Wang, Q.; Zhao, G.; Yao, W.; Xia, Q. Improved oral absorption of (-)-epigallocatechin-3-gallate via self-double-emulsifying solid formulation. Eur. J. Lipid Sci. Technol. 2016, 118, 1115-1124. [CrossRef]

69. Smith, A.J.; Kavuru, P.; Arora, K.K.; Kesani, S.; Tan, J.; Zaworotko, M.J.; Shytle, R.D. Crystal engineering of green tea epigallocatechin-3-gallate (EGCg) cocrystals and pharmacokinetic modulation in rats. Mol. Pharm. 2013, 10, 2948-2961. [CrossRef]

70. ICH. Q2B Validation of Analytical Procedures: Methodology; ICH: Geneva, Switzerland, 1997; Volume 62.

71. Peris-Vicente, J.; Esteve-Romero, J.; Carda-Broch, S. Validation of analytical methods based on chromatographic techniques: An overview. Anal. Sep. Sci. 2015, 1757-1808. [CrossRef]

72. Elkhoudary, M.M.; Abdel Salam, R.A.; Hadad, G.M. Development and optimization of HPLC analysis of metronidazole, diloxanide, spiramycin and cliquinol in pharmaceutical dosage forms using experimental design. J. Chromatogr. Sci. 2016, 54, 1701-1712. [CrossRef]

73. U.S. Food and Drug Administration. Inactive ingredient search for approved drug products. In FDA Database; U.S. Food and Drug Administration: Silver Spring, MD, USA, 2017.

74. U.S. Food and Drug Administration. GRAS Substances (SCOGS) Database; U.S. Food and Drug Administration: Silver Spring, MD, USA, 2012.

75. Gaafar, P.M.; Abdallah, O.Y.; Farid, R.M.; Abdelkader, H. Preparation, characterization and evaluation of novel elastic nano-sized niosomes (ethoniosomes) for ocular delivery of prednisolone. J. Liposome Res. 2014, 24, 204-215. [CrossRef]

76. Jaafar-Maalej, C.; Diab, R.; Andrieu, V.; Elaissari, A.; Fessi, H. Ethanol injection method for hydrophilic and lipophilic drug-loaded liposome preparation. J. Liposome Res. 2010, 20, 228-243. [CrossRef] [PubMed]

77. Farghaly, D.A.; Aboelwafa, A.A.; Hamza, M.Y.; Mohamed, M.I. Topical delivery of fenoprofen calcium via elastic nano-vesicular spanlastics: Optimization using experimental design and in vivo evaluation. AAPS Pharmscitech 2017, 18, 2898-2909. [CrossRef] [PubMed]

78. El Gamal, S.S.; Naggar, V.F.; Allam, A.N. Optimization of acyclovir oral tablets based on gastroretention technology: Factorial design analysis and physicochemical characterization studies. Drug Dev. Ind. Pharm. 2011, 37, 855-867. [CrossRef] [PubMed]

79. Badria, F.A.; Abdelaziz, A.E.; Hassan, A.H.; Elgazar, A.A.; Mazyed, E.A. Development of Provesicular Nanodelivery System of Curcumin as a Safe and Effective Antiviral Agent: Statistical Optimization, In Vitro Characterization, and Antiviral Effectiveness. Molecules 2020, 25, 5668. [CrossRef]

80. Larsson, J. Methods for measurement of solubility and dissolution rate of sparingly soluble drugs. Master's Thesis, Lund University, Lund, Sweden, 2009.

81. Sanna, V.; Singh, C.K.; Jashari, R.; Adhami, V.M.; Chamcheu, J.C.; Rady, I.; Sechi, M.; Mukhtar, H.; Siddiqui, I.A. Targeted nanoparticles encapsulating (-)-epigallocatechin-3-gallate for prostate cancer prevention and therapy. Sci. Rep. 2017, 7, 41573. [CrossRef] 
82. Abd-Elal, R.M.; Shamma, R.N.; Rashed, H.M.; Bendas, E.R. Trans-nasal zolmitriptan novasomes: In-vitro preparation, optimization and in-vivo evaluation of brain targeting efficiency. Drug Deliv. 2016, 23, 3374-3386. [CrossRef]

83. Mali, N.; Darandale, S.; Vavia, P. Niosomes as a vesicular carrier for topical administration of minoxidil: Formulation and in vitro assessment. Drug Deliv. Transl. Res. 2013, 3, 587-592. [CrossRef]

84. Hollands, C. The Animals (scientific procedures) Act 1986. Lancet (Lond. Engl.) 1986, 2, 32. [CrossRef]

85. Kilkenny, C.; Browne, W.J.; Cuthill, I.C.; Emerson, M.; Altman, D.G. Improving bioscience research reporting: The ARRIVE guidelines for reporting animal research. PLoS Biol. 2010, 8, e1000412. [CrossRef]

86. Directive, E. 63/EU of the European Parliament and of the Council of 22 September 2010 on the protection of animals used for scientific purposes. Off. J. Eur. Union 2010, 276, 33-79.

87. Sallam, M.A.; Boscá, M.T.M. Optimization, ex vivo permeation, and stability study of lipid nanocarrier loaded gelatin capsules for treatment of intermittent claudication. Int. J. Nanomed. 2015, 10, 4459. [CrossRef] [PubMed]

88. Jha, S.K.; Karki, R.; Puttegowda, V.D.; Harinarayana, D. In vitro intestinal permeability studies and pharmacokinetic evaluation of famotidine microemulsion for oral delivery. Int. Sch. Res. Not. 2014, 2014, 452051. [CrossRef] [PubMed]

89. Mady, O.Y.; Donia, A.A.; Al-Shoubki, A.A.; Qasim, W. Paracellular pathway enhancement of metformin hydrochloride via molecular dispersion in span 60 microparticles. Front. Pharmacol. 2019, 10, 713. [CrossRef] [PubMed]

90. Al-mahallawi, A.M.; Khowessah, O.M.; Shoukri, R.A. Nano-transfersomal ciprofloxacin loaded vesicles for non-invasive transtympanic ototopical delivery: In-vitro optimization, ex-vivo permeation studies, and in-vivo assessment. Int. J. Pharm. 2014, 472, 304-314. [CrossRef]

91. Bansal, S.; Aggarwal, G.; Chandel, P.; Harikumar, S. Design and development of cefdinir niosomes for oral delivery. J. Pharm. Bioall. Sci. 2013, 5, 318.

92. Gupta, A.; Prajapati, S.K.; Balamurugan, M.; Singh, M.; Bhatia, D. Design and development of a proniosomal transdermal drug delivery system for captopril. Trop. J. Pharm. Res. 2007, 6, 687-693. [CrossRef]

93. Goodlett, C.R.; Stringer, M.; LaCombe, J.; Patel, R.; Wallace, J.M.; Roper, R.J. Evaluation of the therapeutic potential of Epigallocatechin-3-gallate (EGCG) via oral gavage in young adult Down syndrome mice. Sci. Rep. 2020, 10, 1-17. [CrossRef]

94. Lee, S.Y.; Jung, Y.O.; Ryu, J.G.; Oh, H.J.; Son, H.J.; Lee, S.H.; Kwon, J.E.; Kim, E.K.; Park, M.K.; Park, S.H. Epigallocatechin-3-gallate ameliorates autoimmune arthritis by reciprocal regulation of $\mathrm{T}$ helper-17 regulatory $\mathrm{T}$ cells and inhibition of osteoclastogenesis by inhibiting STAT3 signaling. J. Leukoc. Biol. 2016, 100, 559-568. [CrossRef]

95. Sezgin-Bayindir, Z.; Onay-Besikci, A.; Vural, N.; Yuksel, N. Niosomes encapsulating paclitaxel for oral bioavailability enhancement: Preparation, characterization, pharmacokinetics and biodistribution. J. Microencapsul. 2013, 30, 796-804. [CrossRef] 